\title{
Robust Finite-Time Estimation of Biased Sinusoidal Signals: A Volterra Operators Approach
}

\author{
Gilberto Pin ${ }^{a}$, Boli Chen ${ }^{b}$, Thomas Parisini b,c \\ ${ }^{\text {a }}$ Electrolux Italia S.p.A, Italy \\ ${ }^{\mathrm{b}}$ Dept. of Electrical and Electronic Engineering, Imperial College London, UK \\ ${ }^{\mathrm{c}}$ Dept. of Engineering and Architecture, University of Trieste, Italy
}

\begin{abstract}
A novel finite-time convergent estimation technique is proposed for identifying the amplitude, frequency and phase of a biased sinusoidal signal. Resorting to Volterra integral operators with suitably designed kernels, the measured signal is processed yielding a set of auxiliary signals in which the influence of the unknown initial conditions is removed. A second-order sliding mode-based adaptation law - fed by the aforementioned auxiliary signals - is designed for finite-time estimation of the frequency, amplitude, and phase. The worst case behavior of the proposed algorithm in presence of the bounded additive disturbances is fully characterized by Input-to-State Stability arguments. The effectiveness of the estimation technique is evaluated and compared with other existing tools via extensive numerical simulations.
\end{abstract}

Key words: Finite-time estimation; Sinusoidal parameters estimation; Volterra operator, Kernels.

\section{Introduction}

A wide variety of techniques are proposed in the literature to address the frequency estimation problem including extended Kalman filters (see e.g. [18] and the reference cited there in), phase locked loop (PLL) tools (see e.g. [22,31]), adaptive notch filters (ANF) (see e.g. $[20,26])$ and techniques based on the internal model principle [4].

The presence of a bias perturbation affecting the sinusoidal signal has recently generated considerable research efforts. An extension of the so-called EPLL approach presented in [22] has been recently proposed in [21] to address sinusoidal signals affected by a bias term by including an additional integrator in the EPLL algorithm. However, in spite of the popularity of the PLL techniques in electrical power systems applications, typically only local stability properties can be proved or, when averaging analysis is used, global results are available but they are valid only for small adaptation gains (see [31] and [41]).

Email addresses: gilbertopin@alice.it (Gilberto Pin), boli.chen10@imperial.ac.uk (Boli Chen),

t.parisini@gmail.com (Thomas Parisini).
ANF techniques represent an effective alternative to handle the possible bias in the measured signal by introducing an augmented integral loop that makes the modified ANF a frequency-locked-loop (FLL) system (see, [21] and [16]). Resorting to a bank of such FLL filters, multi-sinusoidal estimation problems have also been dealt with in [15]. A variant of the FLL, namely second-order generalized integrator-based orthogonal signal generator (OSG-SOGI) is also exploited in [12] and [13] to cope with a single sinusoidal signal affected by a bias term. In particular, in [13], the frequency adaptive law is integrated into the OSG-SOGI, thus giving rise to a third order generalized integrator-based OSG (OSG-TOGI).

Another important family of frequency estimation methods is based on the state-variable filtering techniques (see e.g., [37]). Specifically, in [2], a fourth-order frequency estimator - characterized by a switched adaptation gain is proposed: this algorithm provides extra attenuation of high-frequency noise in steady state. Recently, an algorithm able to cope with a large class of structured perturbations parametrized in the family of time-polynomial functions has been proposed in [36], that extends the previous work by the authors on the estimation of unbiased harmonic signals (see [35]). The robustness of the method against bounded unstructured perturbations is 
characterized by a Input-to-State-Stability (ISS) analysis. Furthermore, in $[11,8]$, a parallel pre-filtering scheme is presented that extends the single pre-filter used in [35] and [36]. This enhanced structure allows to simplify the adaptation law with respect to [36] and [10], while maintaining the robustness properties with respect to bounded measurement perturbations.

Estimation techniques based on adaptive observers represent a valid alternative to the aforementioned methods and several recent approaches are proposed in the literature. These observer-based methods have been extensively analysed in terms of their stability properties and several papers show that global or semi-global stability can be achieved (see, for example $[25,42,19,3,10,6,7]$ and [9] and the references cited therein). In particular, the very recent paper [9] extends the results presented in [10] (where both structured and unstructured uncertainties are addressed in virtue of suitable pre-filtering techniques) and deals with a 'dual-mode' estimation scheme, incorporating a switching algorithm (depending on the real-time excitation level) into an adaptive observerbased sinusoidal estimator.

While the aforementioned algorithms provide in most cases only asymptotic stability guarantees, there exists a few other methods that are capable to achieve finite-time convergence, , which is a very desirable feature in several application contexts like, for example, micro-grids power systems that are affected by severe frequency fluctuations due to low inertia of generators. For instance, non-asymptotic methods based on algebraic derivatives are proposed in $[39,38]$; however these tools are affected by singularities due to the scalar division based algorithm (this issue has been tackled in $[23,24]$ by using a recursive least squares algorithm, while preserving the deadbeat property). Furthermore, a modulating function-based approach is presented in [14], which allows non-asymptotic frequency detection by suitably-designed modulating functions. The wellknown Prony's method and its many variants represent another class of techniques specialized for estimating the frequency of complex sinusoidal functions (see, for example, [30] and the references cited therein). Recently, the Prony's problem is addressed by an algebraic method reported in [40]. Nevertheless, all the methods currently available lack a theoretical investigation of the finite-time convergence properties in presence of measurement noise.

In this paper, we propose a robust parametric finite-time estimation methodology for biased sinusoidal signals by employing a class of kernel based-linear integral operators, which allow to establish a relationship independent of the unknown initial value of the state of the signal's generator, and in turn yield the adaptation algorithms to identify the sinusoidal parameters in a non-asymptotic way. This is a remarkable result since also in a very recent paper [29], the parameter adaptation scheme de- pends on a relationship which holds only asymptotically, due to the unknown initial error (asymptotic decay), and theoretically prevents the finite-time convergence of the estimates.

In the spirit of prior work presented by the authors on the sole frequency estimation problem (see [33]), this paper deals with a finite-time convergent estimation scheme in which the frequency, amplitude and phase (AFP) of a noisy sinusoidal signal are estimated in finite-time. As shown in the very recent paper [32] dealing with non-asymptotic continuous-time systems identification, Volterra operators induced by suitably defined bivariate kernels, turn out to be an enabling tool for finite-time estimation. In contrast with existing works, the behavior of the estimator in presence of a bounded additive measurement disturbance is rigorously characterized by ISS arguments. To the best of the authors' knowledge, this is the first finite-time convergent sinusoidal estimator the behavior of which is analyzed also in presence of unstructured and bounded measurement perturbations.

The paper is organized as follows: Section 2 introduces several useful notations and basic definitions and provides the problem formulation. In Section 3, the Volterra integral operators are characterized whereas in Section 4, the finite-time estimation technique is illustrated. In Section 5, the stability and robustness properties of the proposed estimation tool are dealt with. Extensive simulation results are provided in Section 6 and Section 7 draws some concluding remarks.

\section{Problem statement and preliminaries}

Consider a biased sinusoidal signal

$$
y(t)=A_{0}+A^{*} \sin (\vartheta(t)), \dot{\vartheta}(t)=\omega^{*}, \vartheta_{0}=\phi
$$

where $A_{0} \in \mathbb{R}_{>0}, A^{*} \in \mathbb{R}_{>0}$ and $\omega^{*} \in \mathbb{R}_{>0}$ are the unknown offset, amplitude and angular frequency, respectively, $\vartheta$ is the instantaneous angle, $\phi$ denote the inital phase shift.

As mentioned in the Introduction, our objective consists in estimating $A^{*}, \omega^{*}$ and $\vartheta$ of the sinusoidal signal (1) (i.e., AFP) within an arbitrarily small finite-time.

For the reader's convenience and for the sake of completeness, let us briefly recall some basic concepts of linear integral operators' algebra (see, for example [5] and the references therein), which are needed to derive the main results presented in the subsequent sections.

In this paper, we use transformations acting on the Hilbert space $\mathcal{L}_{\text {loc }}^{2}\left(\mathbb{R}_{>0}\right)$ of locally square-integrable functions with domain $\mathbb{R}_{>0}$ and range $\mathbb{R}$ (i.e., $u(\cdot) \in$ $\mathcal{L}_{\text {loc }}^{2}\left(\mathbb{R}_{\geq 0}\right) \Leftrightarrow\left(u(\cdot): \mathbb{R}_{\geq 0} \rightarrow \mathbb{R}\right) \wedge\left(\int_{B}|u(t)|^{2} d t<\infty\right.$, 
$\forall$ compact $\left.B \subset \mathbb{R}_{\geq 0}\right)$. Given a function $u \in \mathcal{L}_{\text {loc }}^{2}\left(\mathbb{R}_{\geq 0}\right)$, its image through the Volterra (linear, integral) operator $\mathcal{V}_{K}$ induced by a Hilbert-Schmidt $\mathcal{H} \mathcal{S}$ Kernel Function $K(\cdot, \cdot): \mathbb{R} \times \mathbb{R} \rightarrow \mathbb{R}$ is usually denoted by $\left[\mathcal{V}_{K} u\right](\cdot)$, and is defined by the inner product:

$$
\left[\mathcal{V}_{K} u\right](t) \triangleq \int_{0}^{t} K(t, \tau) u(\tau) d \tau, \quad t \in \mathbb{R}_{\geq 0} .
$$

Any explicit function of time $u(t): t \rightarrow u(t) \in \mathbb{R}$, such that $u(\cdot) \in \mathcal{L}_{\text {loc }}^{2}\left(\mathbb{R}_{\geq 0}\right)$ will be addressed in this paper as a signal. Then, given two scalars $a, b \in \mathbb{R}_{>0}$, with $a<b$, let us denote by $u_{[a, b]}(\cdot)$ and $u_{(a, b]}(\cdot)$ the restriction of a signal $u(\cdot)$ to the closed interval $[a, b]$ and to the left open interval $(a, b]$, respectively. Moreover, let $\mathbf{u}(t) \in \mathbb{R}^{n}, \forall t \geq 0$ be an $i$-times differentiable vector of signals, we denote by $\mathbf{u}^{(1)}$ the vector of the $i$-th order time-derivative signals. Then, we recall the following useful definition:

Definition 1 (Weak (generalized) derivative) Let $u(\cdot) \in \mathcal{L}_{\text {loc }}^{1}\left(\mathbb{R}_{\geq 0}\right)$. We say that $u^{(1)}(\cdot)$ is a weak derivative of $u(\cdot)$ if

$\int_{0}^{t} u(\tau)\left(\frac{d}{d \tau} \phi(\tau)\right) d \tau=-\int_{0}^{t} u^{(1)}(\tau) \phi(\tau) d \tau, \forall t \in \mathbb{R}_{\geq 0}$

for all $\phi \in \mathbb{C}^{\infty}$, with $\phi(0)=\phi(t)=0$.

We remark that $u^{(1)}(\cdot)$ is unique up to a set of zero Lebesgue measure, i.e., it is defined almost everywhere. If $u$ is differentiable in the conventional sense, then its weak derivative is identical to its conventional derivative. Classical rules for the derivation of sum or products of functions also hold for the weak derivative. Given a kernel function $K(\cdot, \cdot)$ in two variables, its $i$-th order weak derivative with respect to the second argument will be denoted as $K^{(i)}, i \in \mathbb{Z}_{\geq 0}$.

For obvious practical implementability reasons, it is convenient to devise a differential form for the operators. By applying the Leibniz differentiation rule to the Volterra integral, the transformed signal $\left[\mathcal{V}_{k} x\right](t)$, for $t \geq 0$, can be obtained as the output of a dynamic system described by the following scalar integro-differential equation:

$$
\begin{aligned}
& \left\{\begin{array}{l}
\dot{\xi}(t)=K(t, t) x(t)+\int_{0}^{t}\left(\frac{\partial}{\partial t} K(t, \tau)\right) x(\tau) d \tau \\
\xi(0)=\xi_{0}=0
\end{array}\right. \\
& {\left[\mathcal{V}_{K} x\right](t)=\xi(t), \forall t \in \mathbb{R}_{>0}}
\end{aligned}
$$

Now, we introduce some useful results dealing with the application of Volterra operators to the derivatives of a signal.
Lemma 2.1 (Volterra image of a signal's derivative) For a given $i \geq 0$, consider a signal $u(\cdot) \in \mathcal{L}^{2}\left(\mathbb{R}_{\geq 0}\right)$ that admits a $i$-th weak derivative in $\mathbb{R}_{>0}$ and a kernel function $K(\cdot, \cdot) \in \mathcal{H S}$ that admits the $i$-th derivative (in the conventional sense) with respect to the second argument, $\forall t \in \mathbb{R}_{\geq 0}$. It holds that:

$$
\begin{aligned}
{\left[\mathcal{V}_{K} u^{(i)}\right](t)=} & \sum_{j=0}^{i-1}(-1)^{i-j-1} u^{(j)}(t) K^{(i-j-1)}(t, t) \\
& +\sum_{j=0}^{i-1}(-1)^{i-j} u^{(j)}(0) K^{(i-j-1)}(t, 0) \\
& +(-1)^{i}\left[\mathcal{V}_{K^{(i)}} u\right](t), \quad \forall t \in \mathbb{R}_{\geq 0}
\end{aligned}
$$

that is, $\left[\mathcal{V}_{K} u^{(i)}\right](\cdot)$ is non-anticipative with respect to $u(\cdot)$ and its first $(i-1)$-th derivatives $u^{(1)}(\cdot), \ldots, u^{(i-1)}(\cdot)$.

Lemma 2.1 (proved in the Appendix) allows to identify a class of kernels such that for each derivative $u^{(i)}, i \in$ $\{0, \cdots, n-1\}$, the image signal $\left[\mathcal{V}_{k} u^{(i)}\right](t), t>0$ is independent from the initial states $u(0), u^{(1)}(0), \cdots, u^{(i-1)}(0)$, according to the following definition.

Definition 2 ( $i$-th order non-asymptotic kernel) Consider a function $K(\cdot, \cdot)$ satisfying the assumptions of Lemma 2.1; if, in addition, for a given $i \geq 1$, the kernel verifies the condition

$$
K^{(j)}(t, 0)=0, \forall t \in \mathbb{R}_{\geq 0}, \forall j \in\{0, \ldots, i-1\},
$$

then, it is called an $i$-th order non-asymptotic kernel.

An instance of the kernel function introduced in Definition 2 will be given in the forthcoming context.

Assuming that $K(\cdot, \cdot)$ is an $n$-th order non-asymptotic kernel function (e.g. BF-NK), then it holds that:

$$
\begin{aligned}
{\left[\mathcal{V}_{K} u^{(i)}\right](t)=} & \sum_{j=0}^{i-1}(-1)^{i-1-j} u^{(j)}(t) K^{(i-j-1)}(t, t) \\
& +(-1)^{i}\left[\mathcal{V}_{K^{(i)}} u\right](t), i \in\{1, \ldots, n-1\}
\end{aligned}
$$

Considering the case $i=1$, from (6) we get

$$
\left[\mathcal{V}_{K} u^{(1)}\right](t)=u(t) K(t, t)-\left[\mathcal{V}_{K^{(1)}} u\right](t) .
$$

Moreover, changing the kernel function $K$ with $K^{(j)}$, for any $j \in\{1, \ldots, n-1\}$ we have that also the following integral equation holds

$$
\left[\mathcal{V}_{K^{(j)}} u^{(i+1)}\right](t)=u^{(i)}(t) K^{(j)}(t, t)-\left[\mathcal{V}_{K^{(j+1)}} u^{(i)}\right](t)
$$


3 Bivariate Feedthrough Non-asymptotic Kernels

Definition 3 ( $i$-th Order BF-NK) [34] A kernel $K(\cdot, \cdot) \in \mathcal{H S}$ that satisfies the assumptions given in Lemma 2.1 and that, for a given $i \geq 1$, also verifies the conditions

$$
K^{(j)}(t, t) \neq 0, \forall t \neq 0, \forall j \in\{0, \ldots, i-1\}
$$

is called i-th Order Bivariate Feedthrough Nonasymptotic (BF-NK) kernel.

Here, we introduce a BF-NK that fulfills (9):

$$
F(t, \tau)=e^{-\beta(t-\tau)}\left(1-e^{-\bar{\beta} \tau}\right)^{n},
$$

which is parametrized by the constants $\beta \in \mathbb{R}_{>0}$ and $\bar{\beta} \in \mathbb{R}_{>0}$. In view of (10), all the non-asymptoticity conditions up to the $n$-th order are met thanks to the factor $\left(1-e^{-\bar{\beta} \tau}\right)^{n}$ regardless of the choice of $\beta$ and $\bar{\beta}$.

For any $i \in\{0,1, \cdots, n\}$, the $\tau$ derived kernel functions read:

$$
F^{(i)}(t, \tau)=e^{-\beta t} \frac{d^{i}}{d \tau^{i}} e^{\beta \tau}\left(1-e^{-\bar{\beta} \tau}\right)^{n} .
$$

Specializing (3) to the kernel (11) with respect to the sinusoidal signal $y(t)$, we have that the transformed signal $\left[\mathcal{V}_{F^{(i)}} y\right](t)$, for any $i \in\{1,2, \cdots, n\}$ can be obtained as the output of a linear time-varying scalar system. Letting $\xi(t)=\left[\mathcal{V}_{F^{(i)}} y\right](t)$, we have that

$$
\begin{aligned}
\dot{\xi}(t) & =F^{(i)}(t, t) y(t)+\int_{0}^{t}\left(\frac{\partial}{\partial t} F^{(i)}(t, \tau)\right) y(\tau) d \tau \\
& =F^{(i)}(t, t) y(t)-\beta \xi(t), t \in \mathbb{R}_{\geq 0}
\end{aligned}
$$

with $\xi(0)=\xi_{0}=0$. Being $F^{(i)}(t, t)$ bounded and $\beta$ strictly positive, it holds that the scalar dynamical system realization of the Volterra operators induced by the proposed kernels is BIBO stable with respect to $y$.

\section{Finite-time AFP estimation in presence of bias}

It is worth noting that the biased sinusoidal signal $y(t)$ given in (1) can be thought as generated by a linear dynamical system governed by the following differential equation:

$$
y^{(3)}(t)=-\Omega^{*} y^{(1)}(t)
$$

where $\Omega^{*}=\omega^{* 2}$. Taking the Volterra linear integral operator on both sides of (13), we obtain

$$
\left[\mathcal{V}_{K} y^{(3)}\right](t)=-\Omega^{*}\left[\mathcal{V}_{K} y^{(1)}\right](t) .
$$

In view of (8), we can expand both sides of (14) by

$$
\begin{aligned}
& {\left[\mathcal{V}_{K} y^{(3)}\right](t)=y^{(2)}(t) K(t, t)-\left[\mathcal{V}_{K^{(1)}} y^{(2)}\right](t)} \\
& =\left[\mathcal{V}_{K^{(2)}} y^{(1)}\right](t)-y^{(1)}(t) K^{(1)}(t, t)+y^{(2)}(t) K(t, t) \\
& =y(t) K^{(2)}(t, t)-\left[\mathcal{V}_{K^{(3)}} y\right](t) \\
& \quad-y^{(1)}(t) K^{(1)}(t, t)+y^{(2)}(t) K(t, t)
\end{aligned}
$$

and

$$
\left[\mathcal{V}_{K} y^{(1)}\right](t)=y(t) K(t, t)-\left[\mathcal{V}_{K^{(1)}} y\right](t) .
$$

After some algebra, we get

$$
\begin{gathered}
{\left[\mathcal{V}_{K^{(3)}} y\right](t)-K^{(2)}(t, t) y(t)+K^{(1)}(t, t) y^{(1)}(t)} \\
-K(t, t) y^{(2)}(t)+\Omega^{*}\left(\left[\mathcal{V}_{K^{(1)}} y\right](t)-K(t, t) y(t)\right)=0 .
\end{gathered}
$$

Consider three BF-NKs (10) denoted by $F_{1}, F_{2}$ and $F_{3}$ with $n=3$

$$
F_{h}(t, \tau)=e^{-\beta_{h}(t-\tau)}\left(1-e^{-\bar{\beta} \tau}\right)^{3}
$$

where $\beta_{h} \in \mathbb{R}_{>0}, \forall h=\{1,2,3\}$ and $\bar{\beta}$ are set by the designers such that

$$
\beta_{i} \neq \beta_{j} \text {, for } i \neq j \text {. }
$$

Then let us rewrite (15) with respect to $F_{1}, F_{2}$ and $F_{3}$, obtaining the following three equations:

$$
\begin{aligned}
& {\left[\mathcal{V}_{F_{1}{ }^{(3)}} y\right](t)-F_{1}^{(2)}(t, t) y(t)+F_{1}^{(1)}(t, t) y^{(1)}(t)} \\
& -F_{1}(t, t) y^{(2)}(t)+\Omega^{*}\left(\left[\mathcal{V}_{F_{1}^{(1)}} y\right](t)-F_{1}(t, t) y(t)\right)=0 \text {, } \\
& {\left[\mathcal{V}_{F_{2}(3)} y\right](t)-F_{2}^{(2)}(t, t) y(t)+F_{2}^{(1)}(t, t) y^{(1)}(t)} \\
& -F_{2}(t, t) y^{(2)}(t)+\Omega^{*}\left(\left[\mathcal{V}_{F_{2}^{(1)}} y\right](t)-F_{2}(t, t) y(t)\right)=0 \text {, } \\
& {\left[\mathcal{V}_{F_{3}\left({ }^{(3)}\right.} y\right](t)-F_{3}^{(2)}(t, t) y(t)+F_{3}^{(1)}(t, t) y^{(1)}(t)} \\
& -F_{3}(t, t) y^{(2)}(t)+\Omega^{*}\left(\left[\mathcal{V}_{F_{3}^{(1)}} y\right](t)-F_{3}(t, t) y(t)\right)=0 \text {. }
\end{aligned}
$$


Now, it is convenient to introduce the following auxiliary signals: ${ }^{1}$

$$
\begin{aligned}
\kappa_{a, h}(t) & \triangleq\left[\mathcal{V}_{F_{h}^{(3)}} y\right](t)-F_{h}^{(2)}(t, t) y(t) \\
\kappa_{b, h}(t) & \triangleq F_{h}^{(1)}(t, t) \\
\kappa_{c, h}(t) & \triangleq-F_{h}(t, t) \\
\kappa_{d, h}(t) & \triangleq\left[\mathcal{V}_{F_{h}^{(1)}} y\right](t)-F_{h}(t, t) y(t), \forall h=1,2,3 .
\end{aligned}
$$

In view of (16), it is worth noting that

$$
F_{1}(t, t)=F_{2}(t, t)=F_{3}(t, t), \forall t \geq 0
$$

which implies that

$$
\kappa_{c, 1}(t)=\kappa_{c, 2}(t)=\kappa_{c, 3}(t), \forall t \geq 0
$$

In the following, we show that the signals $\left[\mathcal{V}_{F_{h}{ }^{(3)}} y\right](t)$ and $\left[\mathcal{V}_{F_{h}^{(1)}} y\right](t)$ appearing in $\kappa_{a, h}(t)$ and $\kappa_{d, h}(t)$, respectively, can be obtained as the output of a linear timevarying system. To this end, let us define the internal state vector

$$
\boldsymbol{\xi}(t) \triangleq\left[\boldsymbol{\xi}_{\beta_{1}}(t)^{\top}, \boldsymbol{\xi}_{\beta_{2}}(t)^{\top}, \boldsymbol{\xi}_{\beta_{3}}(t)^{\top}\right]^{\top} \in \mathbb{R}^{6}
$$

with $\boldsymbol{\xi}_{\beta_{h}}(t) \in \mathbb{R}^{2}, \forall h \in\{1,2,3\}$ in turn defined as

$$
\boldsymbol{\xi}_{\beta_{h}}(t) \triangleq\left[\left[\mathcal{V}_{F_{h}^{(1)}} y\right](t),\left[\mathcal{V}_{F_{h}^{(3)}} y\right](t)\right]^{\top}
$$

In view of (12), the time evolution of the observer's internal state vector $\boldsymbol{\xi}(t)$ is described by

$$
\dot{\boldsymbol{\xi}}(t)=\mathbf{G}_{\xi} \boldsymbol{\xi}(t)+\mathbf{E}_{\xi}(t) y(t),
$$

with $\boldsymbol{\xi}(0)=0$ and where $\mathbf{G}_{\xi} \in \mathbb{R}^{6 \times 6}$ is a diagonal, time invariant and Hurwitz matrix, defined by $\mathbf{G}_{\xi}=\operatorname{blockdiag}\left[\mathbf{G}_{\xi_{1}}, \mathbf{G}_{\xi_{2}}, \mathbf{G}_{\xi_{3}}\right]$, with $\mathbf{G}_{\xi_{h}}=$ $\operatorname{diag}\left(-\beta_{h}\right) \in \mathbb{R}^{2 \times 2}$, while the time-varying input matrices $\mathbf{E}_{\xi}(t) \in \mathbb{R}^{6}$ can be expressed as

$$
\mathbf{E}_{\xi}(t)=\left[\begin{array}{c}
\mathbf{E}_{\xi_{1}}(t) \\
\mathbf{E}_{\xi_{2}}(t) \\
\mathbf{E}_{\xi_{3}}(t)
\end{array}\right]
$$

\footnotetext{
1 It is worth noting that the signals (19) are either kerneldependent (and thus a priori known functions of time) or computable by processing the measurement $y(t)$ by the Volterra operators.
}

where $\mathbf{E}_{\xi_{h}}(t) \in \mathbb{R}^{2}$ are given by:

$$
\mathbf{E}_{\xi_{h}}(t)=\left[\begin{array}{c}
F_{h}^{(1)}(t, t) \\
F_{h}^{(3)}(t, t)
\end{array}\right]
$$

Finally, $\kappa_{a, h}(t)$ and $\kappa_{d, h}(t)$ can be expressed in terms of the scalar elements of $\boldsymbol{\xi}(t)$ :

$$
\begin{aligned}
& \kappa_{a, h}(t) \triangleq \xi_{2 h}(t)-F_{h}^{(2)}(t, t) y(t) \\
& \kappa_{d, h}(t) \triangleq \xi_{2 h-1}(t)-F_{h}(t, t) y(t), \forall h=1,2,3 .
\end{aligned}
$$

Substituting (19) into (18a)-(18c), after some algebra we can eliminate the variables, $y^{(1)}(t)$ and $y^{(2)}(t)$ from the system, obtaining equation (24) that has $\Omega^{*}$ as the only unknown (for brevity, we have dropped the dependence of all variables on $t)$ :

$$
\begin{aligned}
& \kappa_{a, 3} \kappa_{b, 2} \kappa_{c, 1}^{2}-\kappa_{a, 3} \kappa_{b, 1} \kappa_{c, 1} \kappa_{c, 2}+\kappa_{a, 1} \kappa_{b, 3} \kappa_{c, 1} \kappa_{c, 2} \\
& -\kappa_{a, 2} \kappa_{b, 3} \kappa_{c, 1}^{2}+\kappa_{a, 2} \kappa_{b, 1} \kappa_{c, 1} \kappa_{c, 3}-\kappa_{a, 1} \kappa_{b, 2} \kappa_{c, 1} \kappa_{c, 3} \\
& +\Omega^{*}\left(\kappa_{b, 3} \kappa_{c, 1} \kappa_{c, 2} \kappa_{d, 1}-\kappa_{b, 3} \kappa_{c, 1}^{2} \kappa_{d, 2}\right. \\
& +\kappa_{b, 1} \kappa_{c, 1} \kappa_{c, 3} \kappa_{d, 2}-\kappa_{b, 2} \kappa_{c, 1} \kappa_{c, 3} \kappa_{d, 1} \\
& \left.\quad+\kappa_{b, 2} \kappa_{c, 1}^{2} \kappa_{d, 3}-\kappa_{b, 1} \kappa_{c, 1} \kappa_{c, 2} \kappa_{d, 3}\right)=0
\end{aligned}
$$

Let us rearrange the left-hand-side of (24) by using the relationship (21) and by extracting the variables indexed by $a$ and $d$ respectively, thereby getting

$$
\begin{gathered}
\kappa_{a, 1}\left(\kappa_{b, 3}-\kappa_{b, 2}\right)+\kappa_{a, 2}\left(\kappa_{b, 1}-\kappa_{b, 3}\right)+\kappa_{a, 3}\left(\kappa_{b, 2}-\kappa_{b, 1}\right) \\
+\Omega^{*}\left(\kappa_{d, 1}\left(\kappa_{b, 3}-\kappa_{b, 2}\right)+\kappa_{d, 2}\left(\kappa_{b, 1}-\kappa_{b, 3}\right)\right. \\
\left.+\kappa_{d, 3}\left(\kappa_{b, 2}-\kappa_{b, 1}\right)\right)=0
\end{gathered}
$$

which, in turn, can be rearranged in vector form as follows:

$$
\boldsymbol{\kappa}_{a}^{\top}(t) \mathcal{F}(t, t)=-\Omega^{*} \boldsymbol{\kappa}_{d}^{\top}(t) \mathcal{F}(t, t),
$$

with $\boldsymbol{\kappa}_{a}(t)=\left[\begin{array}{lll}\kappa_{a, 1} & \kappa_{a, 2} & \kappa_{a, 3}\end{array}\right]^{\top}, \boldsymbol{\kappa}_{d}(t)=\left[\begin{array}{lll}\kappa_{d, 1} & \kappa_{d, 2} & \kappa_{d, 3}\end{array}\right]^{\top}$ and

$$
\mathcal{F}(t, t)=\left[\begin{array}{c}
\mathcal{F}_{1}(t, t) \\
\mathcal{F}_{2}(t, t) \\
\mathcal{F}_{3}(t, t)
\end{array}\right]=\left[\begin{array}{c}
\kappa_{b, 3}-\kappa_{b, 2} \\
\kappa_{b, 1}-\kappa_{b, 3} \\
\kappa_{b, 2}-\kappa_{b, 1}
\end{array}\right]
$$

that depends only on the kernels $F_{h}(t, t), h=1,2,3$ in correspondence with $\kappa_{b, h}(t)$ and $\kappa_{c, h}(t)$ defined in (19). By rewriting (25) in a compact form, we finally obtain the following equality:

$$
\kappa_{1}(t)=-\Omega^{*} \kappa_{2}(t)
$$

with $\kappa_{1}(t)=\boldsymbol{\kappa}_{a}^{\top}(t) \mathcal{F}(t, t), \kappa_{2}(t)=\boldsymbol{\kappa}_{d}^{\top}(t, t) \mathcal{F}(t)$.

Note that, due to the positivity of the squared frequency, it also holds that:

$$
\left|\kappa_{1}(t)\right|=\Omega^{*}\left|\kappa_{2}(t)\right|
$$


Applying to both sides of (27) the linear operator $V_{K_{g}}$, with kernel $K_{g}(t, \tau)=e^{-g(t-\tau)}, g \in \mathbb{R}_{>0}$, we have that

$$
\Omega^{*}\left[\mathcal{V}_{K_{g}}\left|\kappa_{2}(t)\right|\right](t)=\left[\mathcal{V}_{K_{g}}\left|\kappa_{1}(t)\right|\right](t) .
$$

Defining $\gamma_{i}(t) \triangleq\left[\mathcal{V}_{K_{g}}\left|\kappa_{i}(t)\right|\right](t), i=1,2$, it follows that $\gamma_{1}(t)$ and $\gamma_{2}(t)$ obey the differential equations:

$$
\begin{aligned}
& \dot{\gamma}_{1}(t)=\left|\kappa_{1}(t)\right|-g \gamma_{1}(t) \\
& \dot{\gamma}_{2}(t)=\left|\kappa_{2}(t)\right|-g \gamma_{2}(t)
\end{aligned}
$$

with $\gamma_{1}(0)=\gamma_{2}(0)=0$. Finally, in view of (28) we have that $\Omega^{*}$ verifies the following constraint for any $t$ : $\gamma_{1}(t)=\gamma_{2}(t) \Omega^{*}$. When an estimate $\hat{\Omega}(t)$ of the squared frequency is available, we have that the constraint is not met when $\hat{\Omega} \neq \Omega^{*}$ and a residual term can be introduced:

$$
R_{\Omega}(t)=\gamma_{1}(t)-\gamma_{2}(t) \hat{\Omega}(t) .
$$

The following technical result (the proof is reported in the Appendix) characterizes a persistency of excitation (PE) on signal $\kappa_{2}$ that will be used in the following to devise a sliding mode-based adaptation law for frequency estimation. $^{2}$

Lemma 4.1 (Persistency of Excitation) Given the sinusoidal measurement $y(t)$ and the designed kernels (16), for any sinusoidal signal with non-zero amplitude, there exists $\epsilon \in \mathbb{R}_{>0}$ and $t_{\epsilon} \in \mathbb{R}_{>0}$ such that

$$
\frac{1}{t_{\epsilon}} \int_{t-t_{\epsilon}}^{t}\left|\kappa_{2}(\tau)\right| d \tau \geq \epsilon, \forall t \geq t_{\epsilon}
$$

Owing to (29) and (30), the signal $\gamma_{2}(t)$ (driven by $\left.\left|\kappa_{2}(t)\right|\right)$ is ensured to be positive for all $t \geq t_{\epsilon}$, as shown below:

$$
\begin{aligned}
\gamma_{2}(t) & \geq \int_{t-t_{\epsilon}}^{t} e^{-g(t-\tau)}\left|\kappa_{2}(\tau)\right| d \tau \\
& \geq e^{-g t_{\epsilon}} \int_{t-t_{\epsilon}}^{t}\left|\kappa_{2}(\tau)\right| d \tau \\
& \geq t_{\epsilon} \epsilon e^{-g t_{\epsilon}} .
\end{aligned}
$$

Let $\delta_{\epsilon} \triangleq t_{\epsilon} \epsilon e^{-g t_{\epsilon}}$, then the following adaptation law,

\footnotetext{
2 The PE condition introduced in this paper is a slightly modified version of the classical one available in the literature (see, for instance, [2])
}

exploiting the residual signal $R(\hat{\Omega}, t)$, is proposed:

$\dot{\hat{\Omega}}(t)=\left\{\begin{array}{c}\gamma_{2}(t)^{-1}\left(\eta_{\Omega}(t)+L_{1} \sqrt{\left|R_{\Omega}(t)\right|} \operatorname{sign}\left(R_{\Omega}(t)\right)\right. \\ \left.-\hat{\Omega}(t) \dot{\gamma}_{2}(t)+\dot{\gamma}_{1}(t)\right), \text { if } \gamma_{2}(t) \geq \delta_{\epsilon}, \\ 0, \quad \text { otherwise. }\end{array}\right.$ $\dot{\eta}_{\Omega}(t)=L_{2} \operatorname{sign}\left(R_{\Omega}(t)\right)$

with $\hat{\Omega}(0)>0$ set arbitrarily and $\eta_{\Omega}(0)=0, L_{1}, L_{2} \in$ $\mathbb{R}_{>0}$ are constant gains set by the designer. The system (32) with $\operatorname{sign}(\cdot)$ is understood in the Filippov sense [17].

Later, in Section 5, we will show that the frequency adaptation algorithm (32) is able to identify the squaredfrequency in finite-time in a noise-free scenario, while the estimation error is ISS in presence of measurement noise. Compared to the preliminary work [33] that exploits a first order sliding mode-based adaptation law, the use of a second order sliding mode (see [27] and [44]) allows a significant suppression of the chattering phenomenon, which will be highlighted in the simulation results provided in Section 6. Moreover, the time-based switching condition in [33] is enhanced by a more robust switching mechanism that depends on a known signal and the above excitation condition.

Now, with the aim of estimating the amplitude of the sinusoid in finite time, we will exploit a further structural constraint verified by the derivatives of the measured signal. Indeed, thanks to $(18 \mathrm{a}) \div(18 \mathrm{c})$ and introducing the signals

$$
\rho_{h}\left(\Omega^{*}, t\right)=\kappa_{a, h}(t)+\Omega^{*} \kappa_{d, h}(t), h=1,2,3,
$$

it follows that the derivatives verify the following identities:

$$
\left\{\begin{array}{l}
y^{(1)}(t)=\frac{F_{1}(t, t) \rho_{2}\left(\Omega^{*}, t\right)-F_{2}(t, t) \rho_{1}\left(\Omega^{*}, t\right)}{F_{1}^{(1)}(t, t) F_{2}(t, t)-F_{1}(t, t) F_{2}^{(1)}(t, t)} \\
y^{(2)}(t)=\frac{F_{1}^{(1)}(t, t) \rho_{2}\left(\Omega^{*}, t\right)-F_{2}^{(1)}(t, t) \rho_{1}\left(\Omega^{*}, t\right)}{F_{1}^{(1)}(t, t) F_{2}(t, t)-F_{1}(t, t) F_{2}^{(1)}(t, t)}
\end{array}\right.
$$

if $F_{1}^{(1)}(t, t) F_{2}(t, t)-F_{1}(t, t) F_{2}^{(1)}(t, t) \neq 0$, and

$$
\left\{\begin{aligned}
y^{(1)}(t) & =\frac{F_{1}(t, t) \rho_{3}\left(\Omega^{*}, t\right)-F_{3}(t, t) \rho_{1}\left(\Omega^{*}, t\right)}{F_{1}^{(1)}(t, t) F_{3}(t, t)-F_{1}(t, t) F_{3}^{(1)}(t, t)} \\
y^{(2)}(t) & =\frac{F_{1}^{(1)}(t, t) \rho_{3}\left(\Omega^{*}, t\right)-F_{3}^{(1)}(t, t) \rho_{1}\left(\Omega^{*}, t\right)}{F_{1}^{(1)}(t, t) F_{3}(t, t)-F_{1}(t, t) F_{3}^{(1)}(t, t)}
\end{aligned}\right.
$$

if $F_{1}^{(1)}(t, t) F_{2}(t, t)-F_{1}(t, t) F_{2}^{(1)}(t, t)=0$. The above expressions are always well-posed (singularity-free) for any 
$t>0$ if

$$
\begin{aligned}
& F_{1}^{(1)}(t, t) F_{2}(t, t)-F_{1}(t, t) F_{2}^{(1)}(t, t) \neq \\
& \quad F_{1}^{(1)}(t, t) F_{3}(t, t)-F_{1}(t, t) F_{3}^{(1)}(t, t), \quad \forall t>0 .
\end{aligned}
$$

In the following, we show that the relationship (35) holds for any $t>0$. Thanks to $(20)$, we have

$$
F_{1}^{(1)}(t, t) F_{2}(t, t)=F_{1}^{(1)}(t, t) F_{3}(t, t) .
$$

According to the following implications

$$
\begin{aligned}
& F_{2}^{(1)}(t, t)-F_{3}^{(1)}(t, t)=\left(\beta_{2}-\beta_{3}\right)\left(1-e^{-\bar{\beta} t}\right)^{3} \neq 0, \\
& \Longrightarrow F_{2}^{(1)}(t, t) \neq F_{3}^{(1)}(t, t), \quad \forall t>0, \\
& \Longrightarrow F_{1}(t, t) F_{2}^{(1)}(t, t) \neq F_{1}(t, t) F_{3}^{(1)}(t, t), \quad \forall t>0,
\end{aligned}
$$

the condition (35) is immediately verified.

By deriving twice (1) with respect to time, we have that the true squared-frequency $\Omega^{*}$ verifies the following structural constraint:

$$
\Omega^{*} y^{(1)}(t)^{2}+y^{(2)}(t)^{2}=A^{* 2} \Omega^{* 2} .
$$

Applying to both sides of (36) the linear operator $V_{K_{g_{a}}}$, with kernel $K_{g_{a}}(t, \tau)=e^{-g_{a}(t-\tau)}, g_{a} \in \mathbb{R}_{>0}$, we have that

$$
\left[\mathcal{V}_{K_{g_{a}}} \sqrt{\Omega^{*} y^{(1)}(t)^{2}+y^{(2)}(t)^{2}}\right](t)=A^{*}\left[\mathcal{V}_{K_{g}} \Omega^{*}\right](t)
$$

When only the estimates $\hat{\Omega}(t)$ of $\Omega, \hat{A}(t)$ of $A^{*}$, and $\widehat{y^{(i)}}(t)$ of $y^{(i)}(t)$ are available (the estimated derivatives can be obtained from (33), (34) by using $\hat{\Omega}(t)$ in place of $\Omega^{*}$, thus becoming exact in finite time after $\hat{\Omega}$ has converged to $\Omega^{*}$ ), we have that the constraint (37) is not met in general unless $\hat{A}(t)=A^{*}$, in general, so that we can introduce a time-varying residual that depends on the frequency estimate $\hat{\Omega}(t)$ and on the estimates $\widehat{y^{(1)}}(t)$ and $\widehat{y^{(2)}}(t)$ obtained by (33) and (34):

$$
\begin{aligned}
R_{A}(t) \triangleq\left[\mathcal{V}_{K_{g_{a}}}\right. & \left.\sqrt{\left.\left.\hat{\Omega}(t) \widehat{\left(y^{(1)}\right.}(t)\right)^{2}+\widehat{\left(y^{(2)}\right.}(t)\right)^{2}}\right](t) \\
& -\hat{A}(t)\left[\mathcal{V}_{K_{g}} \hat{\Omega}(t)\right](t) \\
= & \gamma_{A_{1}}(t)-\hat{A}(t) \gamma_{A_{2}}(t), \forall t \geq t_{\epsilon}+\bar{T}_{\Omega}
\end{aligned}
$$

in which $\bar{T}_{\Omega}$ is the upper bound on finite time of convergence for system (32) defined in (45)

$$
\begin{aligned}
& \dot{\gamma}_{A_{1}}(t)=\psi(t)\left(\sqrt{\left.\hat{\Omega}\left(\widehat{y^{(1)}}(t)\right)^{2}+\widehat{\left(y^{(2)}\right.}(t)\right)^{2}}-g_{a} \gamma_{A_{1}}(t)\right), \\
& \dot{\gamma}_{A_{2}}(t)=\psi(t)\left(\hat{\Omega}(t)-g_{a} \gamma_{A_{2}}(t)\right),
\end{aligned}
$$

with $\gamma_{A_{1}}(0)=\gamma_{A_{2}}(0)=0, \psi(t)$ a binary on-off switching signals: $\psi(t)=1, \forall t \geq t_{\epsilon}+\bar{T}_{\Omega}, \psi(t)=0, \forall t<t_{\epsilon}+\bar{T}_{\Omega}$. The following adaptation law based on the second order sliding mode can be designed

$$
\begin{aligned}
& \dot{\hat{A}}(t)=\left\{\begin{array}{r}
\gamma_{A_{2}}(t)^{-1}\left(\eta_{A}(t)+L_{3} \sqrt{\left|R_{A}(t)\right|} \operatorname{sign}\left(R_{A}(t)\right)\right. \\
\left.-\hat{A}(t) \dot{\gamma}_{A_{2}}(t)+\dot{\gamma}_{A_{1}}(t)\right), \forall t>t_{\epsilon}+\bar{T}_{\Omega}, \\
0, \quad \text { otherwise. }
\end{array}\right. \\
& \dot{\eta}_{A}(t)=L_{4} \operatorname{sign}\left(R_{A}(t)\right)
\end{aligned}
$$

with $\hat{A}(t)=\hat{A}(0)=0, \forall t \leq t_{\epsilon}+\bar{T}_{\Omega}$ and where $L_{3}, L_{4} \in$ $\mathbb{R}_{>0}$ are tuning gains set by the designer to steer the residual term $R_{A}(t)$ to 0 in finite-time. Note that the invertibility of $\gamma_{A_{2}}(t)$ is verified for any $t>t_{\epsilon}+\bar{T}_{\Omega}$ due to the positiveness of $\hat{\Omega}(t)$. Finally, the phase of the sinusoidal signal can be easily estimated as follows:

$$
\hat{\vartheta}(t)=\angle\left[\widehat{y^{(2)}}(t)+j \hat{\omega}(t) \widehat{y^{(1)}}(t)\right], \forall t \geq t_{\epsilon}+\bar{T}_{\Omega} .
$$

where $j$ denotes the complex imaginary unit.

\section{Finite-time convergence and robustness anal- ysis in presence of bounded measurement noise}

In this section, we first address the convergence properties of the proposed estimator in the absence of external perturbations. Subsequently, the stability properties in the case of noisy measurements are analyzed.

\section{$5.1 \quad$ Noise-free scenario}

The main result consists in the following theorem.

Theorem 5.1 Given the sinusoidal signal $y(t)$, the estimated frequency $\hat{\Omega}(t)$ that is governed by the adaptation law given by (32) converges to the true value $\Omega^{*}$ in finite time.

Proof. Assuming that the noise-free measurement $y(t)$ is available, we show that the residuals $R_{\Omega}$ and $R_{A}$ converge to zero in finite-time. Thanks to inequality (30) given in Lemma 4.1, the finite-time convergence of the residuals implies also the deadbeat convergence of $\hat{\Omega}(t)$ and $\hat{A}(t)$ to $\Omega^{*}$ and $A^{*}$, respectively. The dynamics of $R_{\Omega}(t)$ obeys the following differential equation:

$$
\dot{R}(\hat{\Omega}, t)=\dot{\gamma}_{1}(t)-\dot{\gamma}_{2}(t) \hat{\Omega}(t)-\gamma_{2}(t) \dot{\hat{\Omega}}(t)
$$

For $t \geq t_{\epsilon}$, by substituting the adaptation law in (40), we have

$$
\dot{R}_{\Omega}(t)=-\eta_{\Omega}(t)-L_{1} \sqrt{\left|R_{\Omega}(t)\right|} \operatorname{sign}\left(R_{\Omega}(t)\right) .
$$


Consider an auxiliary variable vector $\boldsymbol{\zeta}(t)=\left[\zeta_{1}(t), \zeta_{2}(t)\right]^{\top}$ where $\zeta_{1}(t)=\sqrt{\left|R_{\Omega}(t)\right|} \operatorname{sign}\left(R_{\Omega}(t)\right), \zeta_{2}(t)=\eta_{\Omega}(t)$. Then:

$$
\dot{\boldsymbol{\zeta}}(t)=\frac{1}{\sqrt{\left|R_{\Omega}(t)\right|}} \mathbf{M} \boldsymbol{\zeta}(t), \forall R_{\Omega}(t) \neq 0
$$

where

$$
\mathbf{M}=\left[\begin{array}{cc}
-\frac{1}{2} L_{1} & -\frac{1}{2} \\
L_{2} & 0
\end{array}\right]
$$

with spectrum $\lambda\{\mathbf{M}\}=\frac{-L_{1} \pm \sqrt{L_{1}^{2}-8 L_{2}}}{4}$. Being $\mathbf{M}$ Hurwitz, there always exists a positive symmetric matrix $\mathbf{P}$ that solves the linear Lyapunov equation $\mathbf{M}^{\top} \mathbf{P}+\mathbf{P M}=$ $-2 q \mathbf{I}$. Now, let us introduce a quadratic function $V_{\Omega}=$ $\boldsymbol{\zeta}^{\top} \mathbf{P} \boldsymbol{\zeta}$, which can be expanded as

$$
\begin{aligned}
V_{\Omega}(t)=p_{11} \mid & R_{\Omega}(t) \mid+p_{22} \eta_{\Omega}(t)^{2} \\
& +2 p_{12} \sqrt{\left|R_{\Omega}(t)\right|} \operatorname{sign}\left(R_{\Omega}(t)\right) \eta_{\Omega}(t),
\end{aligned}
$$

with $p_{i j}$ the components of the matrix $\mathbf{P}$. Note that, by taking the limit

$$
\lim _{\zeta_{1} \rightarrow 0} V_{\Omega}(\boldsymbol{\zeta})=p_{22} \eta_{\Omega}(t)^{2}
$$

we can rewrite (43) in the following way:

$$
V_{\Omega}(\boldsymbol{\zeta})=\left\{\begin{array}{ll}
\boldsymbol{\zeta}^{\top} \mathbf{P} \boldsymbol{\zeta}, & \text { if } \zeta_{1}(t) \neq 0 \\
p_{22} \zeta_{2}^{2}, & \text { if } \zeta_{1}(t)=0
\end{array} .\right.
$$

After some algebra, the time-derivative of $V_{\Omega}(t)$ along the trajectories of the system (42) can be written as:

$$
\dot{V}_{\Omega}(t)= \begin{cases}-\frac{2 q}{\sqrt{\left|R_{\Omega}(t)\right|}}|\boldsymbol{\zeta}(t)|^{2}, & \text { if } \zeta_{1}(t) \neq 0 \\ 0, & \text { if } \zeta_{1}(t)=0 .\end{cases}
$$

Clearly, $\zeta_{1}(t)$ will stay in 0 when it crosses zero. In case $\zeta_{1}(t) \neq 0$, using the facts that $\left|R_{\Omega}(t)\right|^{-1 / 2}=\left|\zeta_{1}(t)\right|^{-1}>$ $|\boldsymbol{\zeta}(t)|^{-1}$ and $V_{\Omega}(t) \leq \bar{p}|\boldsymbol{\zeta}(t)|^{2}$ with $\bar{p} \triangleq \max \operatorname{eig}(\mathbf{P})$, $\dot{V}_{\Omega}(t)$ can be bounded as

$$
\begin{aligned}
\dot{V}_{\Omega}(t) & \leq-\frac{2 q}{|\boldsymbol{\zeta}(t)|}|\boldsymbol{\zeta}(t)|^{2} \\
& \leq-\frac{2 q}{\sqrt{\bar{p}}} V_{\Omega}(t)^{\frac{1}{2}}
\end{aligned}
$$

In view of the Lyapunov-based finite-time convergence result presented in [1], then (44) implies that $\boldsymbol{\zeta}(t) \rightarrow 0$ with a guaranteed reaching-time $T_{\Omega}\left(V_{\Omega}\left(t_{\epsilon}\right)\right)$ verifying the inequality:

$$
T_{\Omega}\left(V_{\Omega}\left(t_{\epsilon}\right)\right) \leq \frac{\sqrt{V_{\Omega}\left(t_{\epsilon}\right) \bar{p}}}{q} \triangleq \bar{T}_{\Omega} .
$$

Finally, noting that $\zeta(t) \rightarrow 0$ implies $R_{\Omega}(t) \rightarrow 0$, then we can conclude that $\hat{\Omega}(t) \rightarrow \Omega^{*}$ in finite-time.

In the noise-free condition, the frequency estimate coincides with the true frequency, i.e. $\hat{\Omega}(t)=\Omega^{*}$ for all $t>t_{\epsilon}+T_{\Omega}\left(V_{\Omega}\left(t_{\epsilon}\right)\right)$, which makes it possible to prove the finite-time convergence of the estimated amplitude $\hat{A}(t)$ ruled by (38) to $A^{*}$. By defining $\boldsymbol{\zeta}_{A}(t)=\left[\sqrt{\left|R_{A}(t)\right|} \operatorname{sign}\left(R_{A}(t)\right), \eta_{A}(t)\right]^{\top}$, then

$$
\dot{\boldsymbol{\zeta}}_{A}(t)=\frac{1}{\sqrt{\left|R_{A}(t)\right|}} \mathbf{M}_{A} \boldsymbol{\zeta}_{A}(t), \forall R_{A} \neq 0
$$

where

$$
\mathbf{M}_{A}=\left[\begin{array}{cc}
-\frac{1}{2} L_{3} & -\frac{1}{2} \\
L_{4} & 0
\end{array}\right]
$$

Consider a positive symmetric matrix $P_{A}$ that solves the Lyapunov equation $\mathbf{M}_{A}^{\top} \mathbf{P}_{A}+\mathbf{P}_{A} \mathbf{M}_{A}=-2 q_{A} \mathbf{I}$. The convergence time verifies the upper bound:

$$
T_{A}\left(V_{A}\left(t_{\epsilon}+T_{\Omega}\left(V_{\Omega}\left(t_{\epsilon}\right)\right)\right) \leq \frac{\sqrt{V_{A}\left(t_{\epsilon}+T_{\Omega}\left(V_{\Omega}\left(t_{\epsilon}\right)\right)\right) \bar{p}_{A}}}{q_{A}} .\right.
$$

where $\bar{p}_{A} \triangleq \max \operatorname{eig}\left(\mathbf{P}_{A}\right)$. Thus, the finite time convergence of the amplitude estimate occurs at time:

$$
t_{\epsilon}+T_{\Omega}\left(V_{\Omega}\left(t_{\epsilon}\right)\right)+T_{A}\left(V_{A}\left(t_{\epsilon}+T_{\Omega}\left(V_{\Omega}\left(t_{\epsilon}\right)\right)\right)\right) .
$$

This result can be proved by following the same steps taken to prove the finite-time convergence of the frequency estimate and is therefore omitted for the sake of brevity.

Remark 1 Note that the square root term $\sqrt{\left|R_{\Omega}(t)\right|}$ and $\sqrt{\left|R_{A}(t)\right|}$ respectively appear in the adaptation laws (32) and (38) can be replaced by any power within $(0,1)$ without violating the finite-time convergence property. Since a detailed discussion of this subject is beyond the scope of the present article, the reader is referred to $[1,28]$ for more details.

\subsection{Noisy scenario}

Now, we consider a scenario in which the sinusoidal measurement is corrupted by a norm-bounded additive disturbance $d(t):|d(t)| \leq \bar{d}$, such that $\hat{y}(t) \triangleq y(t)+d(t)$.

Let us rewrite (22) introducing the disturbance term $d(t)$ :

$$
\dot{\hat{\boldsymbol{\xi}}}(t)=\mathbf{G} \hat{\boldsymbol{\xi}}(t)+\mathbf{E}(t)(y(t)+d(t))
$$

and the error $\tilde{\boldsymbol{\xi}}(t)=\hat{\boldsymbol{\xi}}(t)-\boldsymbol{\xi}(t)$. Then, the error dynamics can be expressed by

$$
\dot{\tilde{\boldsymbol{\xi}}}(t)=\mathbf{G} \tilde{\boldsymbol{\xi}}(t)+\mathbf{E}(t) d(t)
$$


Being the matrix G Hurwitz, and $\mathbf{E}(t) d(t)$ bounded, $\tilde{\boldsymbol{\xi}}(t)$ is ISS with respect to $\mathbf{E}(t) d(t)$. Each element of the vector $\tilde{\boldsymbol{\xi}}(t) \triangleq\left[\tilde{\xi}_{1}(t), \tilde{\xi}_{2}(t), \cdots, \tilde{\xi}_{6}(t)\right]$ verifies the inequalities depending on the parity of the index:

$$
\begin{aligned}
& \left|\tilde{\xi}_{2 h-1}(t)\right| \leq e^{-\beta_{h} t_{\epsilon}} \tilde{\xi}_{2 h-1}(0)+\frac{1}{\beta_{h}} \sup _{0 \leq \tau<t}\left|F_{h}^{(1)}(\tau, \tau)\right| \bar{d}, \\
& \forall h \in\{1,2,3\}, \forall t \geq t_{\epsilon} .
\end{aligned}
$$

$$
\begin{gathered}
\left|\tilde{\xi}_{2 h}(t)\right| \leq e^{-\beta_{h} t_{\epsilon}} \tilde{\xi}_{2 h}(0)+\frac{1}{\beta_{h}} \sup _{0 \leq \tau<t}\left|F_{h}^{(3)}(\tau, \tau)\right| \bar{d} \\
\forall h \in\{1,2,3\}, \forall t \geq t_{\epsilon} .
\end{gathered}
$$

Let us denote the upper bound of $\left|\tilde{\xi}_{2 h-1}(t)\right|$ and $\left|\tilde{\xi}_{2 h}(t)\right|$ by $\overline{\tilde{\xi}}_{2 h-1}$ and $\overline{\tilde{\xi}}_{2 h}$, respectively, such that

$$
\begin{aligned}
& \overline{\tilde{\xi}}_{2 h-1} \triangleq e^{-\beta_{h} t_{\epsilon}} \tilde{\xi}_{2 h-1}(0)+\frac{1}{\beta_{h}} \sup _{0 \leq \tau<t}\left|F_{h}^{(1)}(\tau, \tau)\right| \bar{d} \\
& \overline{\tilde{\xi}}_{2 h} \triangleq e^{-\beta_{h} t_{\epsilon}} \tilde{\xi}_{2 h}(0)+\frac{1}{\beta_{h}} \sup _{0 \leq \tau<t}\left|F_{h}^{(3)}(\tau, \tau)\right| \bar{d}
\end{aligned}
$$

From (23), we have error signals $\tilde{\kappa}_{a, h}(t) \triangleq \hat{\kappa}_{a, h}(t)-$ $\kappa_{a, h}(t), \tilde{\kappa}_{d, h}(t) \triangleq \hat{\kappa}_{d, h}(t)-\kappa_{d, h}(t)$ follow that

$$
\begin{aligned}
\left|\tilde{\kappa}_{a, h}(t)\right| & =\left|\tilde{\xi}_{2 h}(t)-F_{h}^{(2)}(t, t) d(t)\right| \\
& \leq \tilde{\tilde{\xi}}_{2 h}+\sup _{0 \leq \tau<t}\left|F_{h}^{(2)}(\tau, \tau)\right| \bar{d} \\
\left|\tilde{\kappa}_{d, h}(t)\right| & =\left|\tilde{\xi}_{2 h-1}(t)-F_{h}(t, t) d(t)\right| \\
& \leq \overline{\tilde{\xi}}_{2 h-1}+\sup _{0 \leq \tau<t} F_{h}(\tau, \tau) \bar{d}
\end{aligned}
$$

and then $\left|\tilde{\kappa}_{1}(t)\right|,\left|\tilde{\kappa}_{2}(t)\right|$ that are bounded by

$$
\begin{aligned}
& \left|\tilde{\kappa}_{1}(t)\right|=\left|\hat{\kappa}_{1}(t)-\kappa_{1}(t)\right|=\left|\tilde{\boldsymbol{\kappa}}_{a}^{\top}(t) \mathcal{F}(t, t)\right| \\
& \leq\left|\tilde{\boldsymbol{\kappa}}_{a}(t)\right|_{1}|\mathcal{F}(t, t)|_{1} \\
& =\left(\sum_{h=1}^{3} \overline{\tilde{\xi}}_{2 h}+\sup _{0 \leq \tau<t}\left|F_{h}^{(2)}(\tau, \tau)\right| \bar{d}\right) \sum_{h=1}^{3} \sup _{0 \leq \tau<t}\left|\mathcal{F}_{h}(\tau, \tau)\right| \\
& \left|\tilde{\kappa}_{2}(t)\right|=\left|\hat{\kappa}_{2}(t)-\kappa_{2}(t)\right|=\left|\tilde{\boldsymbol{\kappa}}_{d}^{\top}(t) \mathcal{F}(t, t)\right| \\
& \leq\left|\tilde{\boldsymbol{\kappa}}_{d}(t)\right|_{1}|\mathcal{F}(t, t)|_{1} \\
& =\left(\sum_{h=1}^{3} \overline{\tilde{\xi}}_{2 h-1}+\sup _{0 \leq \tau<t} F_{h}(\tau, \tau) \bar{d}\right) \sum_{h=1}^{3} \sup _{0 \leq \tau<t}\left|\mathcal{F}_{h}(\tau, \tau)\right|
\end{aligned}
$$

are ISS bounded with respect to $\tilde{\boldsymbol{\xi}}(t)$. Moreover, $\boldsymbol{\xi}(t)$ and the kernels are bounded, thus implying the boundedness of $\kappa_{1}(t), \kappa_{2}(t)$ and $\hat{\kappa}_{1}(t), \hat{\kappa}_{2}(t)$. For the sake of further analysis, let

$$
\begin{array}{r}
\overline{\tilde{\kappa}}_{1}(\bar{d}) \triangleq\left(\sum_{h=1}^{3} \overline{\tilde{\xi}}_{2 h}+\sup _{0 \leq \tau<t}\left|F_{h}^{(2)}(\tau, \tau)\right| \bar{d}\right) \\
\times \sum_{h=1}^{3} \sup _{0 \leq \tau<t}\left|\mathcal{F}_{h}(\tau, \tau)\right|
\end{array}
$$

and

$$
\begin{aligned}
\overline{\tilde{\kappa}}_{2}(\bar{d}) \triangleq\left(\sum_{h=1}^{3} \overline{\tilde{\xi}}_{2 h-1}+\sup _{0 \leq \tau<t}\right. & \left.F_{h}(\tau, \tau) \bar{d}\right) \\
& \times \sum_{h=1}^{3} \sup _{0 \leq \tau<t}\left|\mathcal{F}_{h}(\tau, \tau)\right|
\end{aligned}
$$

From inequality (30), for all $t \geq t_{\epsilon}$, it holds that

$$
\begin{aligned}
\frac{1}{t_{\epsilon}} \int_{t-t_{\epsilon}}^{t}\left|\hat{\kappa}_{2}(\tau)\right| d \tau & \geq \frac{1}{t_{\epsilon}} \int_{t-t_{\epsilon}}^{t}\left|\kappa_{2}(\tau)\right|-\left|\tilde{\kappa}_{2}(\tau)\right| d \tau \\
& \geq \epsilon-\frac{1}{t_{\epsilon}} \int_{t-t_{\epsilon}}^{t}\left|\tilde{\kappa}_{2}(\tau)\right| d \tau \\
& \geq \epsilon-\bar{\kappa}_{2}(\bar{d}) \\
& =\epsilon-\sigma(\bar{d})
\end{aligned}
$$

where

$$
\sigma(\bar{d}) \triangleq \overline{\widetilde{\kappa}}_{2}(\bar{d}) .
$$

Now, consider $\hat{\gamma}_{1}(t)$ and $\hat{\gamma}_{2}(t)$ (i.e., the noisy counterparts of $\left.\gamma_{1}(t), \gamma_{2}(t)\right)$. The following dynamic filters driven by the norm of the noisy auxiliary signals $\left|\hat{\kappa}_{1}(t)\right|$ and $\left|\hat{\kappa}_{2}(t)\right|$, respectively, are introduced:

$$
\begin{aligned}
& \dot{\hat{\gamma}}_{1}(t)=\left|\hat{\kappa}_{1}(t)\right|-g \hat{\gamma}_{1}(t) \\
& \dot{\hat{\gamma}}_{2}(t)=\left|\hat{\kappa}_{2}(t)\right|-g \hat{\gamma}_{2}(t) .
\end{aligned}
$$

By analogy with (31), we have

$$
\begin{aligned}
\hat{\gamma}_{2}(t) & =\int_{t-t_{\epsilon}}^{t} e^{-g(t-\tau)}\left|\hat{\kappa}_{2}(\tau)\right| d \tau \\
& \geq t_{\epsilon} e^{-g t_{\epsilon}}(\epsilon-\sigma(\bar{d})), \forall t \geq t_{\epsilon} .
\end{aligned}
$$

To make $\hat{\gamma}_{2}(t)$ strictly positive for $t \geq t_{\epsilon}$, we impose another constraint on the disturbance bound:

$$
\bar{d}<\sigma^{-1}(\epsilon) .
$$

Let us denote by $\hat{\delta}_{\epsilon} \triangleq t_{\epsilon} e^{-g t_{\epsilon}}(\epsilon-\sigma(\bar{d}))$ the transition threshold that determines the triggering time of the adaptation. Then the following implementable fre- 
quency adaptation law in the noisy case is proposed:

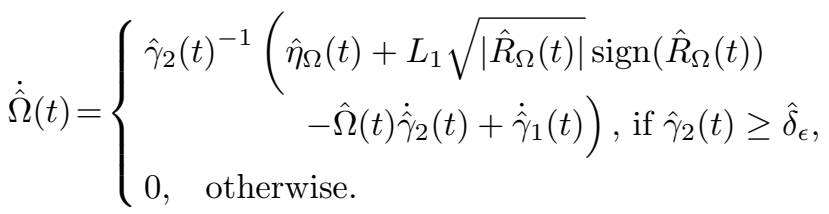

$\dot{\hat{\eta}}_{\Omega}(t)=L_{2} \operatorname{sign}\left(\hat{R}_{\Omega}(t)\right)$

where $\hat{R}_{\Omega}(t)=\hat{\gamma}_{1}(t)-\hat{\gamma}_{2}(t) \hat{\Omega}(t)$.

The following result characterises the stability properties of the adaptive estimation law (50).

Theorem 5.2 Given the sinusoidal signal $y(t)$ and the perturbed measurement $\hat{y}(t)$, the estimated frequency $\hat{\Omega}(t)$ that evolves according to the adaptation law given by (50), enters into a neighborhood of $\Omega^{*}$ in finite-time and the frequency estimation error $\tilde{\Omega}(t)=\hat{\Omega}(t)-\Omega^{*}$ is $I S S$, with respect to any disturbance signal $d(t) \in \mathcal{L}_{\infty}^{1}$ such that $\bar{d}<\sigma^{-1}(\epsilon)$, where $\sigma(\cdot)$ is defined in (48).

Proof. Consider the error variables: $\tilde{\gamma}_{1}(t) \triangleq \hat{\gamma}_{1}(t)-$ $\gamma_{1}(t)$ and $\tilde{\gamma}_{2}(t) \triangleq \hat{\gamma}_{2}(t)-\gamma_{2}(t)$. (29) implies that the dynamics of the error variables obeys the following differential equation:

$$
\dot{\tilde{\gamma}}_{i}(t)=\left|\kappa_{i}(t)+\tilde{\kappa}_{i}(t)\right|-\left|\kappa_{i}(t)\right|-g \tilde{\gamma}_{i}(t), i=1,2
$$

By using the triangle inequality, we obtain

$$
\dot{\tilde{\gamma}}_{i}(t) \leq\left|\tilde{\kappa}_{i}(t)\right|-g \tilde{\gamma}_{i}(t), i=1,2
$$

that, in turn, lead to the following bounds $\forall t \geq t_{\epsilon}$ :

$$
\begin{aligned}
& \left|\tilde{\gamma}_{1}(t)\right| \leq e^{-g t_{\epsilon}} \tilde{\gamma}_{1}(0)+\frac{1}{g} \bar{\kappa}_{1}(\bar{d}), \\
& \left|\tilde{\gamma}_{2}(t)\right| \leq e^{-g t_{\epsilon}} \tilde{\gamma}_{2}(0)+\frac{1}{g} \tilde{\kappa}_{2}(\bar{d}) .
\end{aligned}
$$

Finally, it turns out that $\tilde{\gamma}_{1}(t)$ and $\tilde{\gamma}_{2}(t)$ are ISS with respect to $\tilde{\boldsymbol{\xi}}(t)$ and $\bar{d}$.

Following the same steps taken in the disturbance-free condition, we introduce the noisy counterpart of the auxiliary variable vector $\hat{\boldsymbol{\zeta}}(t)=\left[\hat{\zeta}_{1}(t), \hat{\zeta}_{2}(t)\right]^{\top}$, where $\hat{\zeta}_{1}(t)=\sqrt{\left|\hat{R}_{\Omega}(t)\right|} \operatorname{sign}\left(\hat{R}_{\Omega}(t)\right), \hat{\zeta}_{2}(t)=\hat{\eta}_{\Omega}(t)$, and $\hat{\boldsymbol{\zeta}}(t)$ obeys the differential equations:

$$
\dot{\hat{\boldsymbol{\zeta}}}(t)=\frac{1}{\sqrt{\left|\hat{R}_{\Omega}(t)\right|}} \mathbf{M} \hat{\boldsymbol{\zeta}}(t), \forall \hat{R}_{\Omega}(t) \neq 0
$$

Analogously, the residual $\hat{R}_{\Omega}(t)$ can be proven to remain bounded. From the equality $\hat{R}_{\Omega}(t)=\hat{\gamma}_{1}(t)-\hat{\gamma}_{2}(t) \hat{\Omega}(t)$, we have that

$$
\hat{\Omega}(t)=\frac{\hat{\gamma}_{1}(t)}{\hat{\gamma}_{2}(t)}, \forall t \geq t_{\epsilon}+T_{\Omega}\left(V_{\Omega}\left(t_{\epsilon}\right)\right)
$$

in which $T_{\Omega}\left(V_{\Omega}\left(t_{\epsilon}\right)\right)$ is the time of convergence. It turns out that the proven boundedness of $\hat{\gamma}_{1}(t), \hat{\gamma}_{2}(t)$ and inequality (30) imply the boundedness of $\hat{\Omega}(t)$ for all $t>0$. Moreover, the frequency estimates $\hat{\Omega}(t)$ will enter into the compact region

$$
\begin{array}{r}
\hat{\Omega}(t) \in\left[\inf _{0 \leq \tau<t}\left|\frac{\hat{\gamma}_{1}(\tau)}{\hat{\gamma}_{2}(\tau)}\right|, \sup _{0 \leq \tau<t}\left|\frac{\hat{\gamma}_{1}(\tau)}{\hat{\gamma}_{2}(\tau)}\right|\right] \\
\forall t \geq t_{\epsilon}+T_{\Omega}\left(V_{\Omega}\left(t_{\epsilon}\right)\right) .
\end{array}
$$

which contains $\Omega^{*}$. Hence, the estimation error $\tilde{\Omega}(t)=$ $\hat{\Omega}(t)-\Omega^{*}$ is ISS with respect to $\bar{d}$.

Concerning the amplitude estimation in noisy conditions, analogously to the disturbance-free case, we introduce the perturbed residual signal

$$
\begin{aligned}
\hat{R}_{A}(t) \triangleq\left[\mathcal{V}_{K_{g_{a}}} \sqrt{\left.\left.\left.\hat{\Omega}(t) \widehat{\left(y^{(1)}\right.}(t)\right)^{2}+\widehat{\left(y^{(2)}\right.}(t)\right)^{2}\right](t)}\right. \\
\quad-\hat{A}(t)\left[\mathcal{V}_{K_{g}} \hat{\Omega}(t)\right](t) \\
=\hat{\gamma}_{A_{1}}(t)-\hat{A}(t) \hat{\gamma}_{A_{2}}(t), \quad \forall t \geq t_{\epsilon}+T_{\Omega}\left(V_{\Omega}\left(t_{\epsilon}\right)\right)
\end{aligned}
$$

with

$$
\begin{aligned}
& \dot{\hat{\gamma}}_{A_{1}}(t)=\psi(t)\left(\sqrt{\left.\hat{\Omega}\left(\widehat{y^{(1)}}(t)\right)^{2}+\widehat{\left(y^{(2)}\right.}(t)\right)^{2}}-g_{a} \hat{\gamma}_{A_{1}}(t)\right) \\
& \dot{\hat{\gamma}}_{A_{2}}(t)=\psi(t)\left(\hat{\Omega}(t)-g_{a} \hat{\gamma}_{A_{2}}(t)\right)
\end{aligned}
$$

and $\widehat{y^{(1)}}(t), \widehat{y^{(2)}}(t)$, computed by $(33)-(34)$ with the substitution of $\hat{\rho}_{h}(\hat{\Omega}, t) \triangleq \hat{\kappa}_{a, h}(t)+\hat{\Omega}(t) \hat{\kappa}_{d, h}(t), h=1,2,3$ in place of $\rho_{h}\left(\Omega^{*}, t\right)$.

Then, the implementable amplitude adaptation law in the presence of the disturbance $d(t)$ is given by

$$
\begin{aligned}
& \dot{\hat{A}}(t)=\left\{\begin{array}{r}
\hat{\gamma}_{A_{2}}(t)^{-1}\left(\hat{\eta}_{A}(t)+L_{3} \sqrt{\left|\hat{R}_{A}(t)\right|} \operatorname{sign}\left(\hat{R}_{A}(t)\right)\right. \\
\left.-\hat{A}(t) \dot{\hat{\gamma}}_{A_{2}}(t)+\dot{\hat{\gamma}}_{A_{1}}(t)\right), \forall t>t_{\epsilon}+\bar{T}_{\Omega}, \\
0, \quad \text { otherwise. }
\end{array}\right. \\
& \dot{\hat{\eta}}_{A}(t)=L_{4} \operatorname{sign}\left(R_{A}(t)\right)
\end{aligned}
$$

where $\hat{A}(t)=\hat{A}(0)=0, \forall t \leq t_{\epsilon}+\bar{T}_{\Omega}$. 
Thanks to the boundedness of the $\hat{\Omega}(t), \hat{\kappa}_{a, h}$ and $\hat{\kappa}_{d, h}$, it is straightforward to show that $\widehat{y^{(1)}}(t), \widehat{y^{(2)}}(t)$ are bounded in presence of disturbances. Following the same stability analysis carried out for the frequency estimator, we immediately prove that $\hat{A}(t)$ converges to $\hat{\gamma}_{A_{1}}(t) / \hat{\gamma}_{A_{2}}(t)$ in finite-time.

\section{$6 \quad$ Simulation results}

In this section, extensive numerical examples are given to illustrate the effectiveness of the proposed deadbeat AFP methodology. Several comparisons with other algorithms are included. Moreover, the effect of the discretization algorithm is evaluated in Example 1 comparing Euler and Runge-Kutta tools. In the other examples, Euler discretization is used.

Example 1. Consider the measured sinusoidal signal:

$$
\hat{y}(t)=3 \sin 5 t+d(t)
$$

where $d(t)$ is a zero-mean Gaussian white random disturbance with variance $\sigma^{2}$. We consider a sampling time of $1 \times 10^{-4} \mathrm{~s}$ with 4 -th order Runge-Kutta discretization. The proposed method is equipped with three BF-NKs having parameters: $\beta_{1}=1, \beta_{2}=2, \beta_{3}=3, \bar{\beta}=2.5$ also we let $g=g_{a}=25, L_{1}=30, L_{2}=2, L_{3}=300, L_{4}=5$ and $\delta_{\epsilon}=10^{-5}$. In Fig. 1, the variance of the frequency estimate is compared with the Cramer-Rao bound, showing the effectiveness of the estimator in a noisy scenario (analogous results for the variance of the amplitude and phase estimates can be obtained - not reported for brevity).

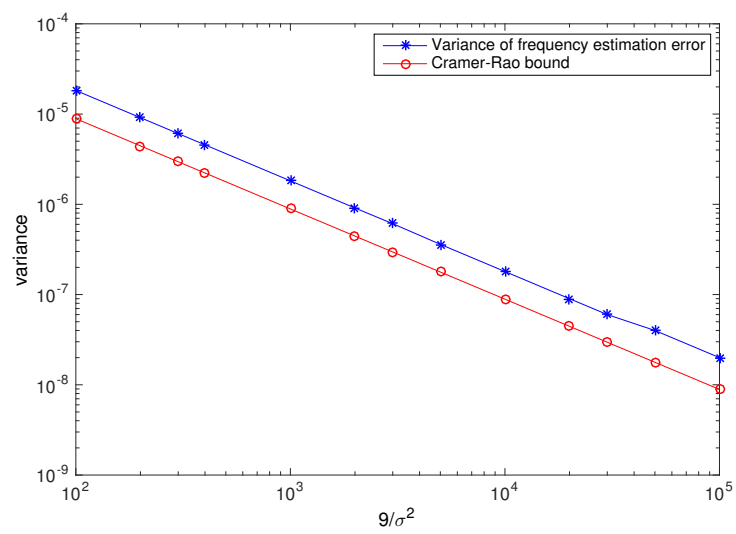

Fig. 1. Comparison of the Cramer-Rao bound and the variance of the frequency estimator on a 30 sample-time-window.

For practical implementation, it is important to analyse the influence of discretization and of the order of the sliding-mode adaptation scheme. This evaluation is made on the biased measured sinusoidal signal:

$$
\hat{y}(t)=2+3 \sin 5 t+d(t),
$$

where $d(t)$ the random noise is uniformly distributed in the interval $[-0.25,0.25]$, and we compare the proposed method with the recent one given in [33] (with the same initial conditions and tuned to show approximately the same initial transient in absence of noise). Results are shown in Fig. 2. The proposed technique shows superior performance compared to the one in [33] when a simple Euler discretization is employed whereas using a Runge-Kutta discretization, the two algorithms show similar performance. It is observed that the chattering phenomenon in steady state is significantly mitigated by using a higher order sliding mode adaptation scheme.

Example 2. In this example, the proposed deadbeat estimator is compared with a classical exponentiallyconverging algorithm, namely the one presented in [42], where the measured noisy signal is:

$$
y(t)=40 \sin \left(100 t+229.18^{\circ}\right)+d(t)
$$

with $d(t)$ denoting a bounded disturbance with uniform distribution in the interval $[-0.5,0.5]$. As shown in Fig. 3, the proposed estimator is characterized by faster convergence, much better transient and steady-state accuracy. As is well known, the finite-time convergence to the residual set of algorithms like [42] can be further reduced at the price of higher noise-sensitivity and larger overshoots. Such a trade-off does not arise in the methodology presented in the paper.

Example 3. In this example, we aim at comparing the proposed deadbeat methodology with two finitetime frequency estimation techniques presented in [29] and [40], To this end, consider the biased signal used in $[29]$ :

$$
y(t)=2+3 \sin (4 t+\pi / 4) .
$$

First, we address the comparison with algorithm [29] whose adaptation law is initialized by $\hat{\Theta}_{1}(0)=[0,0,0]^{\top}$, while the learning gain and the other coefficients are set as $\Gamma_{1}=\operatorname{diag}([50,500,500]), \lambda_{1}=2.5, \lambda_{2}=5, l=$ $1, \kappa=0.001$. Our algorithm method is tuned by $\beta_{1}=$ $1, \beta_{2}=2, \beta_{3}=3, \bar{\beta}=2.5, g=3, L_{1}=30, L_{2}=$ $2, \delta_{\epsilon}=1 \times 10^{-4}$ and the same initial frequency guess $\hat{\Omega}(0)=\sqrt{5}$. The comparison is reported in Fig. 4 . It can be noticed that the proposed method shows a significantly better performance both in noise-free and noisy scenarios (the measurement noise has the same characteristics as in the previous example).

It is worth noting that, the proposed estimation methodology inherently annihilates the effects of the (unknown) initial conditions. To enhance this significant feature, in Fig. 5 simulations referred to different initial conditions in a noise-free scenario and using the same input signal (53) are reported. As can be noticed, unlike algorithm [29], the proposed method yields in finite-time the same estimate of the frequency, irrespective of the initial conditions. 


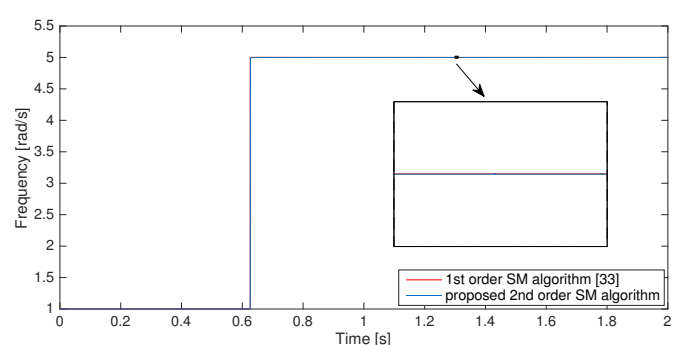

(a)

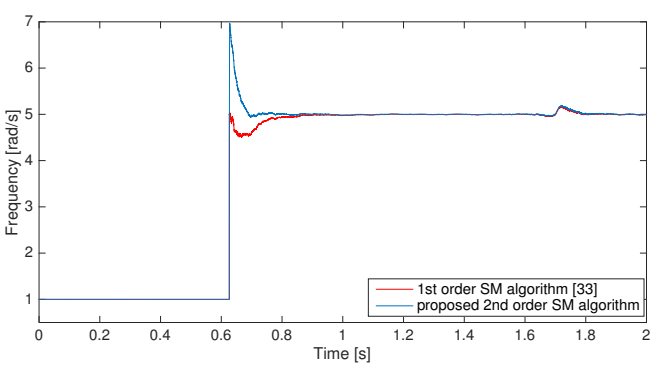

(c)

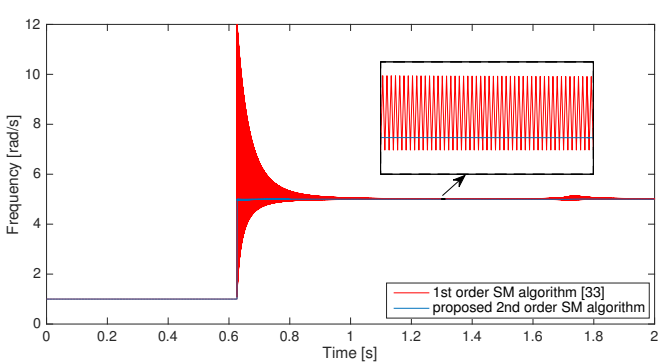

(b)

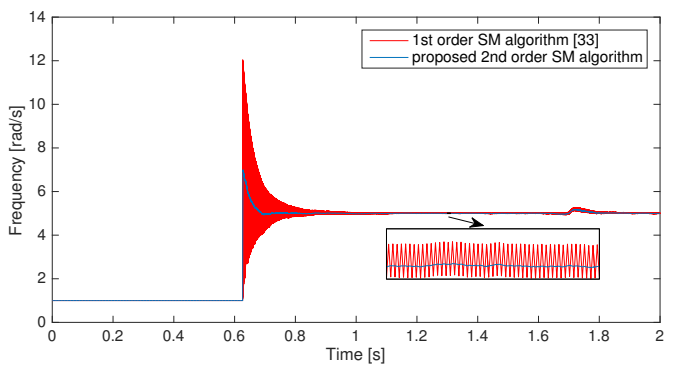

(d)

Fig. 2. Time-behavior of the estimated frequency by using the proposed method and the method [33]: (a) and (b) noise-free scenario with 4-th order Runge-Kutta and Euler discretization, respectively; (c) and (d) noisy scenario with 4-th order Runge-Kutta and Euler discretization, respectively.

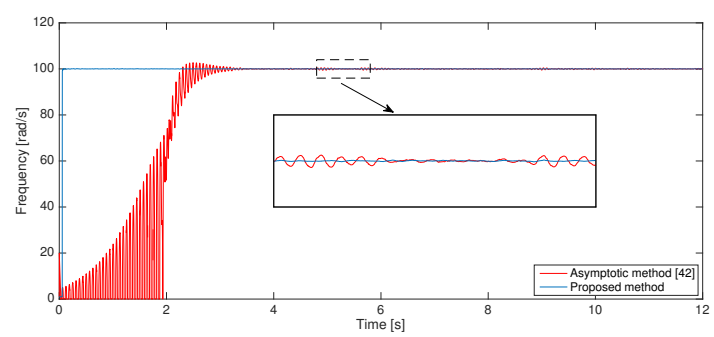

Fig. 3. Time-behavior of the estimated frequency by using the proposed method and the asymptotic method [42] in noisy scenario.

For completeness, in Fig. 6 we show the performance of the amplitude estimator and the behavior of the reconstructed sinusoid to appreciate the accuracy of the phase estimate.

As a second comparison with a finite-time frequency estimation technique, we now consider the algebraic method illustrated in [40]. The sinusoidal input (53) is rewritten as (Prony's problem) $y(t)=$ $2+\frac{3}{2} e^{j(4 t+\pi / 4)}+\frac{3}{2} e^{j(-4 t-\pi / 4)}$ and is processed by the algorithm in [40] with the order $n$ equal to 2 . Both methods are tuned to show a similar transient behavior in the noise-free scenario (see Fig. 7(a)). Under the same conditions, we also compare the behavior of the techniques in the presence of $d(t)$ which is assumed uniformly distributed in $[-0.25,0.25]$. As shown in Fig. 7(b), the algebraic method [40] is susceptible to

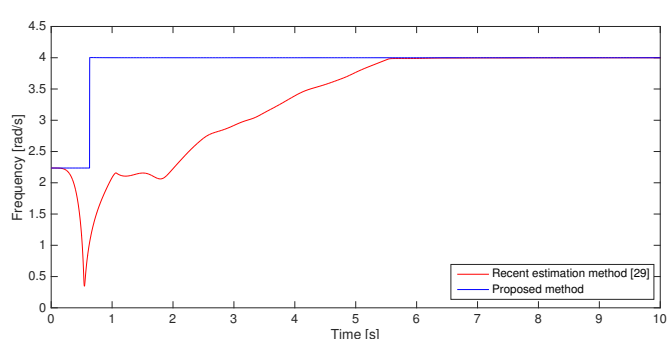

(a)

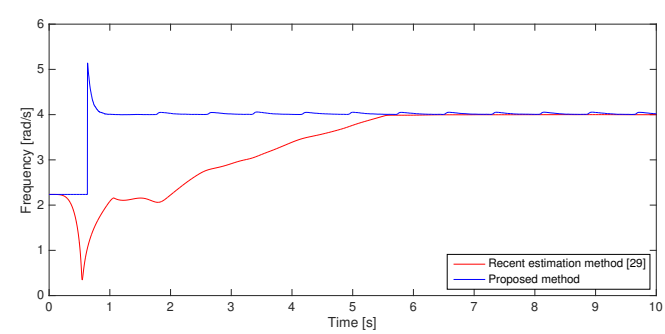

(b)

Fig. 4. Time-behavior of the estimated frequency by using the proposed method (blue line) compared with the time behavior of the estimated frequency obtained by the method [29] (red line): (a) noise-free case; (b) noisy case.

numerical problems in the noisy scenario because of the lack of internal stability.

Example 4. This last comparison example is devoted 


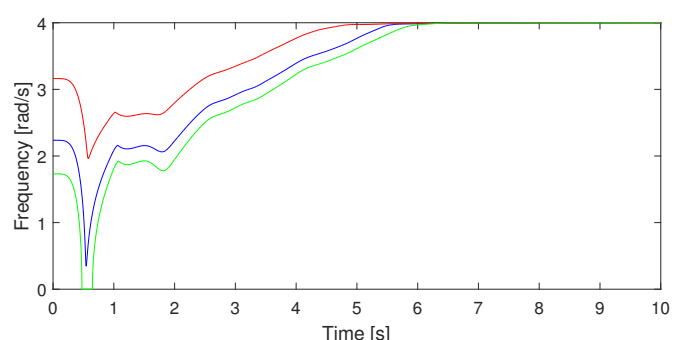

(a)

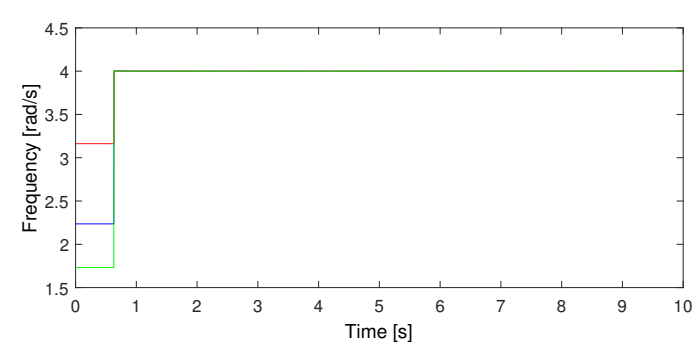

(b)

Fig. 5. Time-behavior of the estimated frequency with different initial conditions: (a) algorithm [29]; (b) proposed algorithm.

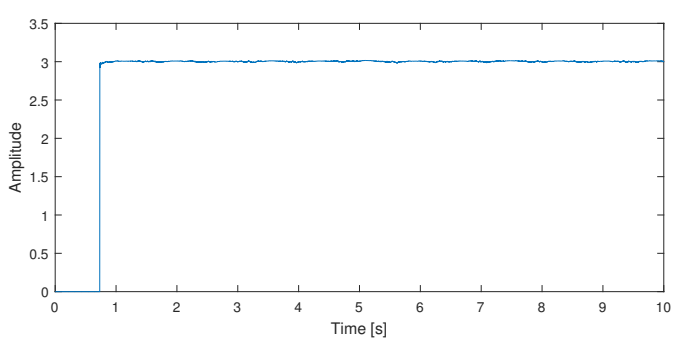

(a)

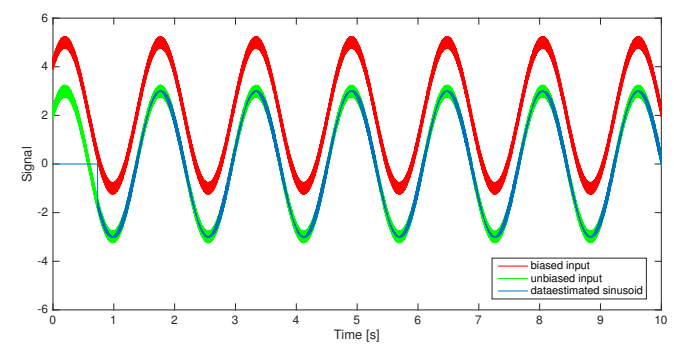

(b)

Fig. 6. (a) Time-behavior of the amplitude estimate; (b) time-behavior of the reconstructed sinusoidal signal.

to apply the proposed deadbeat AFP estimator to the more challenging scenario in which the following biased and noisy sinusoidal signal is considered:

$$
\hat{y}(t)=A_{0}(t)+A(t) \sin (2 \pi f(t) t)+d(t),
$$

where the amplitude, frequency and the offset change

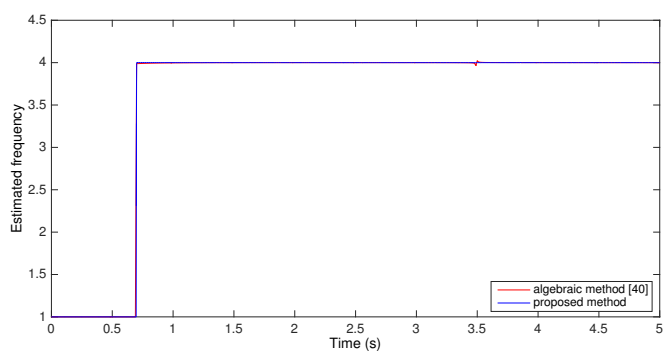

(a)

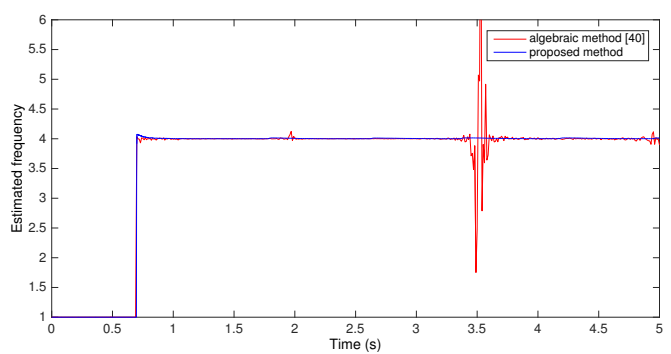

(b)

Fig. 7. Time-behavior of the estimated frequency by using the proposed method (blue line) compared with the time behavior of the estimated frequency obtained by the algebraic method [40] (red line): (a) noise-free case; (b) noisy case.

over time according to the following pattern: $A(t)=$ $10, f(t)=50 \mathrm{~Hz}, A_{0}(t)=1,0 \leq t<0.5, A(t)=$ $12, f(t)=52 \mathrm{~Hz}, A_{0}(t)=0.8,0.5 \leq t<1 . d(t)$ is a randomly generated perturbation with uniform distribution in the interval $[-0.5,0.5]$. The performance of our algorithm is compared with the frequency estimation modulation function technique presented in [14]. The parameters of the algorithm [14] are set as $T=\pi / 10, n=$ $500, \mu=0.99, K=6$. The proposed method is equipped with three kernels having parameters: $\beta_{1}=50, \beta_{2}=$ $80, \beta_{3}=100, \bar{\beta}=60$, while $g=30, g_{a}=100, L_{1}=$ $2 \times 10^{4}, L_{2}=20, L_{3}=1 \times 10^{5}, L_{4}=50$.

Theoretically, the algorithm in [14] identifies the true frequency "almost instantaneously" as it is enabled, whereas the proposed method is theoretically able to achieve finite-time convergence with a "tunable" convergence rate determined by the parameters $L_{1}, L_{2}$. The comparison is shown in Fig. 8. The two algorithms are tuned with similar response to the initial frequency and the same initial condition $\hat{f}(0)=48 \mathrm{~Hz}$ for fair comparison. Although both methods track satisfactorily the frequency step-change, the proposed estimator performs slightly better in terms of robustness against measurement noise.

For completeness, in Fig. 9 we show the performance of the amplitude estimator and the behavior of the reconstructed sinusoid to appreciate the accuracy of the phase estimate (the algorithm in [14] only provides a frequency estimator). 


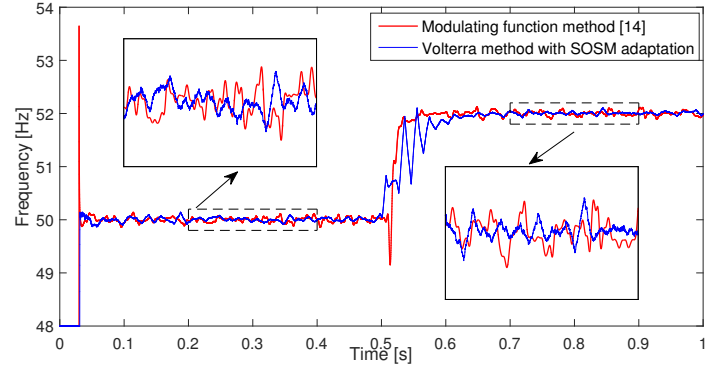

Fig. 8. Time-behavior of the estimated frequency by using the proposed method (blue line) compared with the time behaviors of the estimated frequency by the finite-time method [14] (red line).

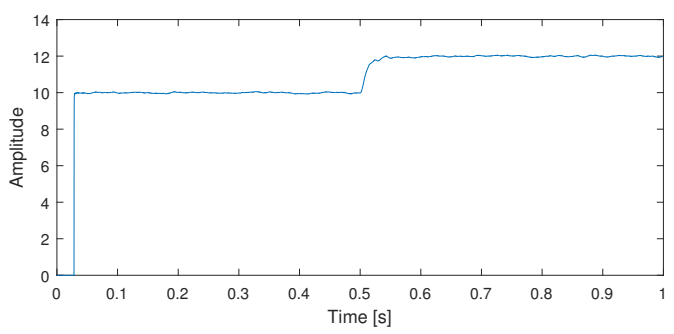

(a)

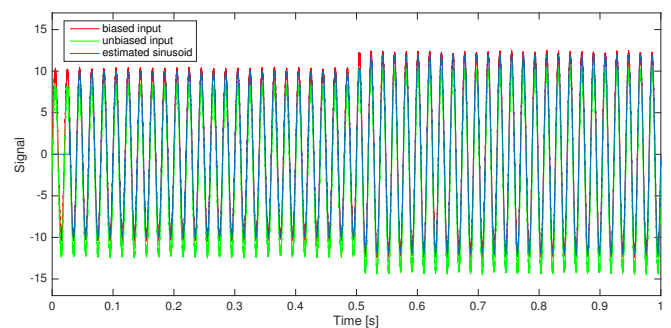

(b)

Fig. 9. (a) Time-behavior of the amplitude estimate; (b) time-behavior of the reconstructed sinusoidal signal.

\section{Concluding remarks}

In this paper, the problem of AFP identification from a noisy and biased measurement has been addressed. A novel estimator is proposed to provide reliable frequency estimates almost instantaneously. The method consists in processing the measured signal with Volterra operators, to obtain auxiliary signals that are used in combination with second-order sliding mode adaptation laws to estimate the frequency, the amplitude and the phase of the original signal. This algorithm has been proved to be finite-time convergent in nominal condition and enjoys ISS stability properties with respect to bounded measurement perturbations. Extensive numerical examples have been reported showing the effectiveness of the proposed method compared to several techniques available in the literature.

\section{References}

[1] S. P. Bhat and D. S. Bernstein. Finite-time stability of continuous autonomous systems. SIAM J. Control and Optimization, 38(3):751-766, 2000.

[2] A. A. Bobtsov, D. Efimov, A. A. Pyrkin, and A. Zolghadri. Switched algorithm for frequency estimation with noise rejection. IEEE Trans. on Automatic Control, 57(9):24002404, 2012

[3] A. A. Bobtsov. New approach to the problem of globally convergent frequency estimator. Int. J. of Adaptive Control and Signal Processing, 22(3):306-317, 2008.

[4] L. J. Brown and Q. Zhang. Periodic disturbance cancellation with uncertain frequency. Automatica, 40(4):631-637, 2004.

[5] T. A. Burton. Volterra Integral and Differential Equations. Elsevier, 2005.

[6] D. Carnevale and A. Astolfi. Hybrid observer for global frequency estimation of saturated signals. IEEE Trans. on Automatic Control, 54(10):2461-2464, 2009.

[7] D. Carnevale and A. Astolfi. A hybrid observer for frequency estimation of saturated multi-frequency signals. In Proc. of the IEEE Conf. on Decision and Control and European Control Conference, p. 2577-2582, Orlando, FL, USA, 2011.

[8] B. Chen, G. Pin, W. M. Ng, S.Y.R. Hui, and T. Parisini. A parallel prefiltering approach for the identification of a biased sinusoid signal: Theory and experiments. Int. Journal of Adaptive Control and Signal Processing, 29(12):1591-1608, 2015.

[9] B. Chen, G. Pin, W. M. Ng, C. K. Lee, S. Y. R. Hui, and T. Parisini. An adaptive observer-based switched methodology for the identification of a perturbed sinusoidal signal: Theory and experiments. IEEE Trans. on Signal Processing, 62(24):6355-6365, 2014.

[10] B. Chen, G. Pin, and T. Parisini. Adaptive observer-based sinusoid identification: structured and bounded unstructured measurement disturbances. In Proc. of the European Control Conference, p. 2645-2650, Zurich, Switzerland, 2013.

[11] B. Chen, G. Pin, and T. Parisini. Robust parametric estimation of biased sinusoidal signals: a parallel pre-filtering approach. In Proc. of the IEEE Conf. on Decision and Control, Los Angeles, p. 1804-1809, 2014.

[12] G. Fedele and A. Ferrise. Non adaptive second order generalized integrator for identification of a biased sinusoidal signal. IEEE Trans. on Automatic Control, 57(7):1838-1842, 2012.

[13] G. Fedele, A. Ferrise, and P. Muraca. An adaptive quasinotch filter for a biased sinusoidal signal estimation. In IEEE International Conference on Control and Automation (ICCA), pages 1060-1065, Santiago, 2011.

[14] G. Fedele and L.Coluccio. A recursive scheme for frequency estimation using the modulating function method. Applied Mathematics and Computation, 216(5):1393-1400, 2010.

[15] G. Fedele and A. Ferrise. A frequency-locked-loop filter for biased multi-sinusoidal estimation. IEEE Trans. on Signal Processing, 62(5):1125-1134, 2014.

[16] G. Fedele, C. Picardi, and D. Sgro. A power electrical signal tracking strategy based on the modulating functions method. IEEE Trans. on Industrial Electronics, 56(10):4079-4087, 2009.

[17] A. F. Filippov. Differential equations with discontinuous right-hand sides. Dordrecht, Boston, London: Kluwer Academic Publishers, 1988. 
[18] H. Hajimolahoseini, M. R. Taban, and H. Soltanian-Zadeh. Extended kalman filter frequency tracker for nonstationary harmonic signals. Measurement, 45(1):126-132, 2012.

[19] M. Hou. Parameter identification of sinusoids. IEEE Trans. on Automatic Control, 57(2):467-472, 2012.

[20] L. Hsu, R. Ortega, and G. Damm. A globally convergent frequency estimator. IEEE Trans. on Automatic Control, 44(4):698-713, 1999.

[21] M. Karimi-Ghartemani, S. Ali Khajehoddin, P. K. Jain, A. Bakhshai, and M. Mojiri. Addressing dc component in PLL and notch filter algorithms. IEEE Trans. on Power Electronics, 27(1):78-86, 2012.

[22] M. Karimi-Ghartemani and A. K. Ziarani. A nonlinear time-frequency analysis method. IEEE Trans. on Signal Processing, 52(6):1585-1595, 2004.

[23] D.Y. Liu, O. Gibaru, and W. Perruquetti. Parameters estimation of a noisy sinusoidal signal with time-varying amplitude. In Proc. 19th Mediterranean conference on Control and automation (MED'11), pages 570 - 575, Corfu, 2011.

[24] A. Luviano-Juarez, J. Cortes-Romero, and H. Sira-Ramirez. Parameter identification of a discretized biased noisy sinusoidal signal. Measurement, 60:129-138, 2015.

[25] R. Marino and P. Tomei. Global estimation of $n$ unknown frequencies. IEEE Trans. on Automatic Control, 47(8):13241328, 2002.

[26] M. Mojiri, D. Yazdani, and A. Bakhshai. Robust adaptive frequency estimation of three-phase power systems. IEEE Trans. on Instrumentation and Measurement, 59(7):1793$1802,2010$.

[27] J. A. Moreno and M. Osorio. Strict Lyapunov functions for the super-twisting algorithm. IEEE Trans. on Automatic Control, 57(4):1035-1040, 2012.

[28] E. Moulay and W. Perruquetti. Finite time stability and stabilization of a class of continuous systems. Journal of Mathematical Analysis and Application, 323(2):1430-1443, 2006.

[29] J. Na, J. Yang, X. Wu, and Y. Guo. Robust adaptive parameter estimation of sinusoidal signals. Automatica, 53:376-384, 2015.

[30] M. R. Osborne and G. K. Smyth. Finite time stability and stabilization of a class of continuous systems. SIAM Journal on Scientific Computing, pages 119-138, 1995.

[31] G. Pin. A direct approach for the frequency-adaptive feedforward cancellation of harmonic disturbances. IEEE Trans. on Signal Processing, 58(7):3513-3530, 2010.

[32] G. Pin, A. Assalone, M. Lovera, and T. Parisini. Non-asymptotic kernel-based parametric estimation of continuous-time linear systems. IEEE Trans. on Automatic Control, 61(2):360-373, 2016.

[33] G. Pin, B. Chen, and T. Parisini. Deadbeat kernel-based frequency estimation of a biased sinusoidal signal. In Proc. of the European Control Conference, Linz, Austria, p. 2937$2944,2015$.

[34] G. Pin, M. Lovera, A. Assalone, and T. Parisini. Kernelbased non-asymptotic state estimation for linear continuoustime system. In Proc. of the American Control Conference, p. 3123 - 3128, Washington, DC, 2013.

[35] G. Pin, T. Parisini, and M. Bodson. Robust parametric identification of sinusoidal signals: an input-to-state stability approach. In Proc. of the IEEE Conf. on Decision and Control, p. 6104-6109, Orlando, FL, 2011.
[36] G. Pin, B. Chen, T. Parisini, and M. Bodson. Robust sinusoid identification with structured and unstructured measurement uncertainties. IEEE Trans. on Automatic Control, 59(6):1588-1593, 2014.

[37] A. A. Pyrkin, A. A. Bobtsov, D. Efimov, and A. Zolghadri. Frequency estimation for periodical signal with noise in finite time. In Proc. of the IEEE Conf. on Decision and Control, p. 3646-3651, Orlando, FL, 2011.

[38] J. R. Trapero, H. Sira-Ramirez, and V. F. Battle. An algebraic frequency estimator for a biased and noisy sinusoidal signal. Signal Processing, 87(6):1188-1201, 2007.

[39] J. R. Trapero, H. Sira-Ramirez, and V. F. Battle. A fast on-line frequency estimator of lightly damped vibrations in flexible structures. Journal of Sound and Vibration, 307(12):365-378, 2007.

[40] R. Ushirobira, W. Perruquetti, M. Mboup, and M. Fliess. Algebraic parameter estimation of a multi-sinusoidal waveform signal from noisy data. In Proc. of the IEEE European Control Conference, Zurich, Switzerland, 2013.

[41] B. Wu and M. Bodson. A magnitude/phase-locked loop approach to parameter estimation of periodic signals. IEEE Trans. on Automatic Control, 48(4):612-618, 2003.

[42] X. Xia. Global frequency estimation using adaptive identifiers. IEEE Trans. on Automatic Control, 47(7):1188$1193,2002$.

[43] K. Yasuda and K. Hirai. Upper and lower bounds on the solution of the algebraic matrix riccati and lyapunov equation. IEEE Trans. on Automatic Control, 24(6):483-487, 1979.

[44] L. Zhao, J. Huang, H. Liu, B. Li, and W. Kong. Second-order sliding-mode observer with online parameter identification for sensorless induction motor drives. IEEE Trans. on Industrial Electronics, 61(10):5280-5289, 2014.

\section{A Proof of Lemma 2.1}

Integrating by parts, we have:

$$
\begin{gathered}
{\left[\mathcal{V}_{K} u^{(i)}\right](t)=\int_{0}^{t} K(t, \tau) u^{(i)}(\tau) d \tau=u^{(i-1)}(t) K(t, t)} \\
-u^{(i-1)}(0) K(t, 0)-\int_{0}^{t} K^{(1)}(t, \tau) u^{(i-1)}(\tau) d \tau . \quad(\text { A. } 1)
\end{gathered}
$$

The integral operator on the right-hand side of (A.1) can be further split by parts:

$$
\begin{aligned}
& -\int_{0}^{t} K^{(1)}(t, \tau) u^{(i-1)}(\tau) d \tau=-u^{(i-2)}(t) K^{(1)}(t, t) \\
& +u^{(i-2)}(0) K^{(1)}(t, 0)+\int_{0}^{t} K^{(2)}(t, \tau) u^{(i-2)}(\tau) d \tau .
\end{aligned}
$$


Proceeding by induction we obtain

$$
\begin{gathered}
\int_{0}^{t} K(t, \tau) u^{(i)}(\tau) d \tau=(-1)^{i} \int_{0}^{t} K^{(i)}(t, \tau) u(\tau) d \tau \\
+\sum_{j=1}^{i}(-1)^{j+1} u^{(i-j)}(t) K^{(j-1)}(t, t) \\
+\sum_{j=1}^{i}(-1)^{j} u^{(i-j)}(0) K^{(j-1)}(t, 0)
\end{gathered}
$$

that is, the function obtained by applying the Volterra operator to the $i$-th derivative is non-anticipative with respect to lower-order derivatives. The identity (4) can be verified by rearranging indexing of the summation in (A.2), thus completing the proof.

\section{B Proof of Lemma 4.1}

Let us recall the definition of $\kappa_{2}(t)$, which can be rewritten as follows:

$$
\begin{aligned}
\kappa_{2}(t)= & {\left[\mathcal{V}_{F_{1}^{(1)}} y\right](t)\left(F_{3}^{(1)}(t, t)-F_{2}^{(1)}(t, t)\right) } \\
& +\left[\mathcal{V}_{F_{2}^{(1)}} y\right](t)\left(F_{1}^{(1)}(t, t)-F_{3}^{(1)}(t, t)\right) \\
& +\left[\mathcal{V}_{F_{3}^{(1)}} y\right](t)\left(F_{2}^{(1)}(t, t)-F_{1}^{(1)}(t, t)\right) .
\end{aligned}
$$

Moreover, consider two kernels $F_{i}, F_{j}$, with $i \neq j$ among the designed kernels $F_{h}, h=1,2,3$. Then, some algebra gives

$$
F_{i}^{(1)}(t, t)-F_{j}^{(1)}(t, t)=\left(\beta_{i}-\beta_{j}\right)\left(1-e^{-\bar{\beta} t}\right)^{3} \neq 0
$$

for all $t>0$ while satisfying constraint (17). Owing to $(22)$, the following condition in the Laplace domain (with zero initial conditions) can be written:

$$
\mathscr{L}\left\{\left[\mathcal{V}_{F_{h}^{(1)}} y\right](t)\right\}=\frac{1}{s+\beta_{h}} \mathscr{L}\left\{F_{h}^{(1)}(t, t) y(t)\right\}
$$

where

$$
\begin{aligned}
F_{h}^{(1)}(t, t)=\beta_{h}-3\left(\beta_{h}-\bar{\beta}\right) e^{-\bar{\beta} t} & +3\left(\beta_{h}-2 \bar{\beta}\right) e^{-2 \bar{\beta} t} \\
& -\left(\beta_{h}-3 \bar{\beta}\right) e^{-3 \bar{\beta} t}
\end{aligned}
$$

Since $y(t)$ takes on the sinusoidal form (1), we get

$$
\begin{aligned}
& \mathscr{L}\left\{F_{h}^{(1)}(t, t) y(t)\right\}=\beta_{h} \frac{A_{0}}{s}+\beta_{h} A^{*} \frac{s \sin \phi+\omega \cos \phi}{s^{2}+\omega^{* 2}} \\
- & 3\left(\beta_{h}-\bar{\beta}\right)\left[\frac{A_{0}}{s+\bar{\beta}}+A^{*} \frac{(s+\bar{\beta}) \sin \phi+\omega \cos \phi}{(s+\bar{\beta})^{2}+\omega^{* 2}}\right] \\
+ & 3\left(\beta_{h}-2 \bar{\beta}\right)\left[\frac{A_{0}}{s+2 \bar{\beta}}+A^{*} \frac{(s+2 \bar{\beta}) \sin \phi+\omega \cos \phi}{(s+2 \bar{\beta})^{2}+\omega^{* 2}}\right] \\
- & \left(\beta_{h}-3 \bar{\beta}\right)\left[\frac{A_{0}}{s+3 \bar{\beta}}+A^{*} \frac{(s+3 \bar{\beta}) \sin \phi+\omega \cos \phi}{(s+3 \bar{\beta})^{2}+\omega^{* 2}}\right] .
\end{aligned}
$$

Except for the first two elements in (B.4), the other terms are exponentially decaying to zero. Hence, from (B.3) it follows that, for any choice of $\bar{t}>0$, one gets

$$
\begin{array}{r}
{\left[\mathcal{V}_{F_{h}^{(1)}} y\right](t)=A_{0}+\frac{\beta_{h} A^{*}}{\sqrt{\beta_{h}^{2}+\omega^{* 2}}}} \\
\sin \left(\omega^{*} t+\phi+\phi_{F_{h}}\right) \\
+\mathcal{O}_{1 \bar{t}}(t), \forall t \geq \bar{t}
\end{array}
$$

where $\phi_{F_{h}}=\arctan \left(\omega^{*} / \beta_{h}\right)$ and where $\left|\mathcal{O}_{1 \bar{t}}(t)\right|$ can be made as small as desired by selecting a suitably large value of $\bar{t}$. Analogously, (B.2) can be expressed as

$$
F_{i}^{(1)}(t, t)-F_{j}^{(1)}(t, t)=\beta_{i}-\beta_{j}+\mathcal{O}_{2 \bar{t}}(t), \forall t \geq \bar{t} .
$$

where $\left|\mathcal{O}_{2 \bar{t}}(t)\right|$ can be made as small as desired by selecting a suitably large value of $\bar{t}$. Therefore, for $t \geq \bar{t},(\mathrm{~B} .1)$ can be rewritten as

$$
\begin{gathered}
\kappa_{2}(t)=\frac{\beta_{1} A^{*}}{\sqrt{\beta_{1}^{2}+\omega^{* 2}}} \sin \left(\omega^{*} t+\phi+\phi_{F_{1}}\right)\left(\beta_{3}-\beta_{2}\right) \\
+\frac{\beta_{2} A^{*}}{\sqrt{\beta_{2}^{2}+\omega^{* 2}}} \sin \left(\omega^{*} t+\phi+\phi_{F_{2}}\right)\left(\beta_{1}-\beta_{3}\right) \\
+\frac{\beta_{3} A^{*}}{\sqrt{\beta_{3}^{2}+\omega^{* 2}}} \sin \left(\omega^{*} t+\phi+\phi_{F_{3}}\right)\left(\beta_{2}-\beta_{1}\right)+\mathcal{O}_{\bar{t}}(t),
\end{gathered}
$$

where $\mathcal{O}_{\bar{t}}(t)$ represents a residual whose absolute value can be made arbitrarily small by selecting a suitably large $\bar{t}$.

Let us now show that, for any $t>\bar{t}$, there exists $t_{p}(t) \in$ $(\bar{t}, t)$, such that $\kappa_{2}\left(t_{p}(t)\right) \neq 0$. Suppose ab absurdo that $\nexists t_{p}(t) \in \mathbb{R}_{>\bar{t}}: \kappa_{2}\left(t_{p}(t)\right) \neq 0$, that is, $\kappa_{2}(t)=0, \forall t>\bar{t}$. Consider a time instant $t_{1}: t_{1}>\bar{t}$, such that $\omega^{*} t_{1}+\phi=$ $2 k \pi$, for some $k \in \mathbb{Z}$. Then:

$$
\begin{aligned}
\kappa_{2}\left(t_{1}\right)= & \frac{\beta_{1} A^{*}}{\sqrt{\beta_{1}^{2}+\omega^{* 2}}} \sin \left(\phi_{F_{1}}\right)\left(\beta_{3}-\beta_{2}\right) \\
& +\frac{\beta_{2} A^{*}}{\sqrt{\beta_{2}^{2}+\omega^{* 2}}} \sin \left(\phi_{F_{2}}\right)\left(\beta_{1}-\beta_{3}\right) \\
& +\frac{\beta_{3} A^{*}}{\sqrt{\beta_{3}^{2}+\omega^{* 2}}} \sin \left(\phi_{F_{3}}\right)\left(\beta_{2}-\beta_{1}\right)+\mathcal{O}_{\bar{t}}\left(t_{1}\right),
\end{aligned}
$$


that is $\left(\right.$ since $\left.\sin \left(\phi_{F_{h}}\right)=\frac{\omega^{*}}{\sqrt{\beta_{h}^{2}+\omega^{* 2}}}\right)$ :

$$
\begin{array}{r}
\kappa_{2}\left(t_{1}\right)=\frac{\beta_{1} A^{*} \omega^{*}}{\beta_{1}^{2}+\omega^{* 2}}\left(\beta_{3}-\beta_{2}\right)+\frac{\beta_{2} A^{*} \omega^{*}}{\beta_{2}^{2}+\omega^{* 2}}\left(\beta_{1}-\beta_{3}\right) \\
+\frac{\beta_{3} A^{*} \omega^{*}}{\beta_{3}^{2}+\omega^{* 2}}\left(\beta_{2}-\beta_{1}\right)+\mathcal{O}_{\bar{t}}\left(t_{1}\right) .
\end{array}
$$

Then, $\kappa_{2}\left(t_{1}\right)=0$ if

$$
\begin{aligned}
\frac{\beta_{1} A^{*} \omega^{*}}{\beta_{1}^{2}+\omega^{* 2}}\left(\beta_{3}-\beta_{2}\right)+\frac{\beta_{2} A^{*} \omega^{*}}{\beta_{2}^{2}+\omega^{* 2}}\left(\beta_{1}-\beta_{3}\right) \\
\quad+\frac{\beta_{3} A^{*} \omega^{*}}{\beta_{3}^{2}+\omega^{* 2}}\left(\beta_{2}-\beta_{1}\right)=-\mathcal{O}_{\bar{t}}\left(t_{1}\right) .
\end{aligned}
$$

Next, consider a time instant $t_{2}: t_{2}>\bar{t}$, such that $\omega^{*} t_{2}+$ $\phi=2 k \pi+\pi / 2$, for some $k \in \mathbb{Z}$. Then, similarly as above and owing to the relationship $\cos \left(\phi_{F_{h}}\right)=\frac{\beta_{h}}{\sqrt{\beta_{h}^{2}+\omega^{* 2}}}$, it follows that $\kappa_{2}\left(t_{2}\right)=0$ if

$$
\begin{aligned}
\frac{\beta_{1}^{2} A^{*}}{\beta_{1}^{2}+\omega^{* 2}}\left(\beta_{3}-\beta_{2}\right)+\frac{\beta_{2}^{2} A^{*}}{\beta_{2}^{2}+\omega^{* 2}}\left(\beta_{1}-\beta_{3}\right) \\
+\frac{\beta_{3}^{2} A^{*}}{\beta_{3}^{2}+\omega^{* 2}}\left(\beta_{2}-\beta_{1}\right)=-\mathcal{O}_{\bar{t}}\left(t_{2}\right) .
\end{aligned}
$$

Finally, consider $t_{3}: t_{3}>\bar{t}$, such that $\omega^{*} t_{3}+\phi+\phi_{F_{1}}=$ $2 k \pi$, for some $k \in \mathbb{Z}$. Since $\phi_{F_{h}}$ is the phase shift of a first-order filter, it turns out that $\left|\phi_{F_{i}}-\phi_{F_{j}}\right|<\pi / 2, i \neq$ $j$; hence

$$
\sin \left(\omega^{*} t_{3}+\phi+\phi_{F_{2}}\right) \neq 0
$$

and

$$
\sin \left(\omega^{*} t_{3}+\phi+\phi_{F_{3}}\right) \neq 0 .
$$

Therefore, $\kappa_{2}\left(t_{3}\right)=0$ if

$$
\chi_{2}\left(\beta_{1}-\beta_{3}\right)+\chi_{3}\left(\beta_{2}-\beta_{1}\right)=-\mathcal{O}_{\bar{t}}\left(t_{3}\right),
$$

where

$$
\chi_{2} \triangleq \frac{\beta_{2} A^{*}}{\sqrt{\beta_{2}^{2}+\omega^{* 2}}} \sin \left(\omega^{*} t_{3}+\phi+\phi_{F_{2}}\right)
$$

and

$$
\chi_{3} \triangleq \frac{\beta_{3} A^{*}}{\sqrt{\beta_{3}^{2}+\omega^{* 2}}} \sin \left(\omega^{*} t_{3}+\phi+\phi_{F_{3}}\right) .
$$

Now, from (B.5), (B.6), and (B.7) it follows that a necessary condition for $\kappa_{2}(t)=0, \forall t>\bar{t}$ is $\kappa_{2}\left(t_{1}\right)=\kappa_{2}\left(t_{2}\right)=$ $\kappa_{2}\left(t_{3}\right)=0$, that is:

$$
\left[\begin{array}{ccc}
\frac{\beta_{1} A^{*} \omega^{*}}{\beta_{1}^{2}+\omega^{* 2}} & \frac{\beta_{2} A^{*} \omega^{*}}{\beta_{2}^{2}+\omega^{* 2}} & \frac{\beta_{3} A^{*} \omega^{*}}{\beta_{3}^{2}+\omega^{* 2}} \\
\frac{\beta_{1}^{2} A^{*}}{\beta_{1}^{2}+\omega^{* 2}} & \frac{\beta_{2}^{2} A^{*}}{\beta_{2}^{2}+\omega^{* 2}} & \frac{\beta_{3}^{2} A^{*}}{\beta_{3}^{2}+\omega^{* 2}} \\
0 & \chi_{2} & \chi_{3}
\end{array}\right]\left[\begin{array}{c}
\beta_{3}-\beta_{2} \\
\beta_{1}-\beta_{3} \\
\beta_{2}-\beta_{1}
\end{array}\right]=-\left[\begin{array}{c}
\mathcal{O}_{\bar{t}}\left(t_{1}\right) \\
\mathcal{O}_{\bar{t}}\left(t_{2}\right) \\
\mathcal{O}_{\bar{t}}\left(t_{3}\right)
\end{array}\right] .
$$

For any choice of $\beta_{1}, \beta_{2}, \beta_{3}$ satisfying (17), it is immediate to conclude that there exists a sufficiently large value of $\bar{t}$ such that no selection of $t_{1}, t_{2}, t_{3} \in(\bar{t}, \infty)$ does exist such that the vector equality (B.8) holds. Hence, we can conclude that there always exists a time instant $t_{p}(t) \in(\bar{t}, t)$, such that $\kappa_{2}\left(t_{p}(t)\right) \neq 0$.

Now, consider a time instant $t_{\epsilon}(t) \triangleq t-t_{p}(t)$. From the continuity of function $\kappa_{2}(t)$ is continuous, we immediately get:

$$
\frac{1}{t_{\epsilon}(t)} \int_{t-t_{\epsilon}(t)}^{t}\left|\kappa_{2}(\tau)\right| d \tau=\frac{1}{t-t_{p}(t)} \int_{t_{p}(t)}^{t} \begin{aligned}
& \left|\kappa_{2}(\tau)\right| d \tau>0 \\
& \forall t>t_{p}(t) .
\end{aligned}
$$

Therefore, there exists a strictly positive function $\epsilon(t)$ depending on $\left|\kappa_{2}\left(t_{p}(t)\right)\right|$, such that

$$
\frac{1}{t_{\epsilon}(t)} \int_{t-t_{\epsilon}(t)}^{t}\left|\kappa_{2}(\tau)\right| d \tau \geq \epsilon(t)
$$

which, in turn, implies that the signal $\kappa_{2}$ admits a local $\mathrm{PE}$ condition with a level of excitation $\epsilon(t)$, such that

$$
\frac{1}{t_{\epsilon}(t)} \int_{t-t_{\epsilon}(t)}^{t}\left|\kappa_{2}(\tau)\right| d \tau \geq \epsilon(t), \forall t \geq t_{p}(t)>\bar{t} .
$$

Since (B.9) holds for any $t \in \mathbb{R}_{>\bar{t}}$, one may infer that for all $t$ the global $\mathrm{PE}$ condition (30) holds conservatively with $\epsilon=\inf _{t>\bar{t}} \epsilon(t)$ and $t_{\epsilon}=\sup _{t>\bar{t}} t_{\epsilon}(t)$, thus ending the proof. 